\title{
RECONSTRUCCIÓN MORFOLÓGICA Y FONOLÓGICA DE LOS PRONOMBRES PERSONALES INDEPENDIENTES DEL PROTOATZINCA
}

\author{
Etna T. Pascacio Montijo \\ Universidad Nacional Autónoma de México \\ etnatpm@gmail.com
}

This paper presents a morphological and phonological reconstruction of the personal independent pronouns of the Proto-Atzinca language, on the basis of data from current Matlatzinca and Ocuilteco languages and colonial documents. In all the Atzinca languages we find eleven pronouns as independent words which distinguish first, second and third person; singular, dual and plural number for each person, as well as inclusive and exclusive for the first-person dual and plural pronouns. Each of these categories is marked with different morphemes in the pronominal words. By comparing these words within the Atzinca languages and making the reconstruction for the Proto-Atzinca, we find that there are no morphological changes, but only phonological and phonetic changes.

Keywords: historical linguistics, comparative method, pronouns, matlatzinca, ocuilteco

En este artículo presento la reconstrucción morfológica y fonológica de los pronombres personales independientes del protoatzinca, con base en datos del 
matlatzinca y ocuilteco actuales, y de documentos coloniales. En todas las lenguas atzincas encontramos once pronombres independientes que distinguen primera, segunda y tercera persona, singular, dual y plural para cada persona y exclusividad en los pronombres de primera persona dual y plural. Cada una de estas categorías se expresa con un morfema distinto en las palabras pronominales. Uno de los principales resultados de la comparación y reconstrucción que aquí presento es que no hay cambios morfológicos diacrónicos, pero sí los hay en los niveles fonológico y fonético.

Palabras clave: lingüística histórica, método comparativo, pronombres, matlatzinca, ocuilteco

\section{INTRODUCCIÓN}

En este texto presento una propuesta de reconstrucción morfológica y fonológica de los pronombres personales independientes (abreviados como PPI) del protoatzinca. ${ }^{1}$ En el primer apartado abordo la reconstrucción de las categorías gramaticales que coocurren en los PPI, con sus oposiciones, y presento la jerarquía intracategorial, basándome en Benveniste (1971[1966]), Jakobson (1981[1975]), Siewierska (2004) y en las propuestas del Seminario Permanente sobre Persona Gramatical del Instituto de Investigaciones Antropológicas de la UNAM. ${ }^{2}$ En el segundo

${ }^{1}$ Llamo "lenguas atzincas" a las lenguas registradas o mencionadas con los nombres "matlatzinca", "pirinda" y "ocuilteco" en la época colonial, y a las lenguas matlatzinca y ocuilteca o tlahuica actuales. Llamo "protoatzinca" a la lengua madre de estas lenguas. Utilizo el término "atzinca" como una etiqueta y no por su significado en náhuatl, sino porque, por un lado, hace referencia de forma precisa a la lengua de San Juan Atzingo, como propuso Soustelle (1993[1937]), y por el otro, también remite al termino "matlatzinca", que es el más utilizado para el resto de las lenguas atzincas.

2 Veáse Aguilar y Valiñas (2008), Meléndez (2011) y Pascacio (2011). 
apartado presento la reconstrucción de los PPI de primera y segunda persona, mientras que en el tercero presento la reconstrucción de los PPI de tercera persona. En el último apartado concluyo con una serie de reflexiones derivadas de las reconstrucciones.

Quiero aclarar desde este punto que separo los pronombres de primera y segunda personas de los de tercera, porque tanto en el protoatzinca como en las lenguas actuales tienen una estructura de palabra distinta. Este hecho puede explicarse desde una perspectiva teórica general y se sustenta tipológicamente, ya que de acuerdo con Benveniste (1971[1966]) y Siewierska (2004), podemos esperar que los exponentes para tercera persona se comporten de manera distinta a los de primera y segunda personas.

Asimismo, quiero señalar que el corpus que analicé está formado con datos del matlatzinca y el ocuilteco o tlahuica recopilados en campo y por datos registrados por autores anteriores, incluyendo documentos coloniales. ${ }^{3}$ Para analizar los datos provenientes de otros autores, fue necesario hacer una regularización gráfica, la cual, en el caso de los documentos coloniales, implicó la interpretación fonética de las grafías originales. Metodológicamente parto de considerar una lengua por comunidad o por registro, para fines comparativos. Asimismo, a lo largo del texto hago referencia a cada lengua por el lugar en el que se habla o por el lugar en el que se hicieron los registros, ya que sería confuso llamar a la mayoría "matlatzinca".

3 Actualmente, el matlatzinca se habla solamente en la comunidad de San Francisco Oxtotilpan, municipio de Temascaltepec y el ocuilteco en seis localidades del municipio de Ocuilan, ambos municipios situados en el Estado de México. De acuerdo con el Censo General de Población y Vivienda 2010 del INEGI, hay aproximadamente 750 hablantes de matlatzinca y 700 de ocuilteco. 
Como se sabe, las lenguas atzincas son tonales. Sin embargo, en la reconstrucción que propongo no presento una reconstrucción del tono, ya que no cuento con suficientes datos para todas las lenguas atzincas.

\section{LAS CATEGORÍAS PRONOMINALES DEL PROTOATZINCA}

En los PPI del protoatzinca las oposiciones dentro de las categorías gramaticales de persona, número y exclusividad se marcaban con exponentes morfológicos afijales. En las lenguas hijas encontramos las mismas oposiciones con los mismos exponentes morfológicos, es decir, no hay cambios en las categorías gramaticales del protoatzinca hacia las lenguas hijas. Para entender con mayor claridad la expresión formal de cada una de las oposiciones dentro de cada categoría presento la estructura de palabra morfológica de los PPI en el esquema 1.

Esquema 1. Estructura de palabra morfológica de los PPI del protoatzinca
a) Primera y segunda personas
*[[[RAíz -PERSONA]-NÚMERo]-eXCLUSIVo] ${ }^{4}$
b) Tercera persona
*[R-[TERCERA PERSONA -NÚMERo]]

Los pronombres de primera y segunda persona del protoatzinca estaban compuestos por una raíz pronominal a la cual se le sufijaba un morfema de persona, después se sufijaba un morfema de número y, finalmente, en los pronombres de primera persona no singular se sufijaba el morfema

\footnotetext{
${ }^{4}$ Con el asterisco se indican los elementos reconstruidos.
} 
de exclusivo. Los pronombres de tercera persona estaban formados por el morfema de tercera persona, al cual se sufijaba un morfema de número y después se prefijaba el exponente $\mathrm{R}$. Aún no está definida la categoría o la función del prefijo R, pero más adelante describiré su posición y su comportamiento. A continuación presento las oposiciones expresadas para cada categoría y su jerarquía en los PPI.

Las oposiciones que se presentan en todas las lenguas para la categoría de persona son participante del acto de habla ([+/-PAH]) y subjetividad ([+/SUB]). En el protoatzinca, los pronombres con el rasgo [+PAH] que corresponden a los de primera y segunda personas compartían la misma estructura de palabra, la cual tenía como núcleo morfológico la raíz pronominal ${ }^{*} k a$, mientras que la tercera persona, con el rasgo [-PAH], tenía una estructura de palabra distinta con el exponente de tercera persona como núcleo morfológico ${ }^{*} t^{\prime}$. Dentro de los pronombres con el rasgo [+PAH] la oposición de [+SUB] se expresaba con el sufijo *-ki primera persona', mientras que [-SUB] se expresaba con el sufijo ${ }^{*}-c i c$ 'segunda personà (véase esquema 2 ).

Esquema 2. Exponentes para las oposiciones de la categoría de persona en los PPI del protoatzinca

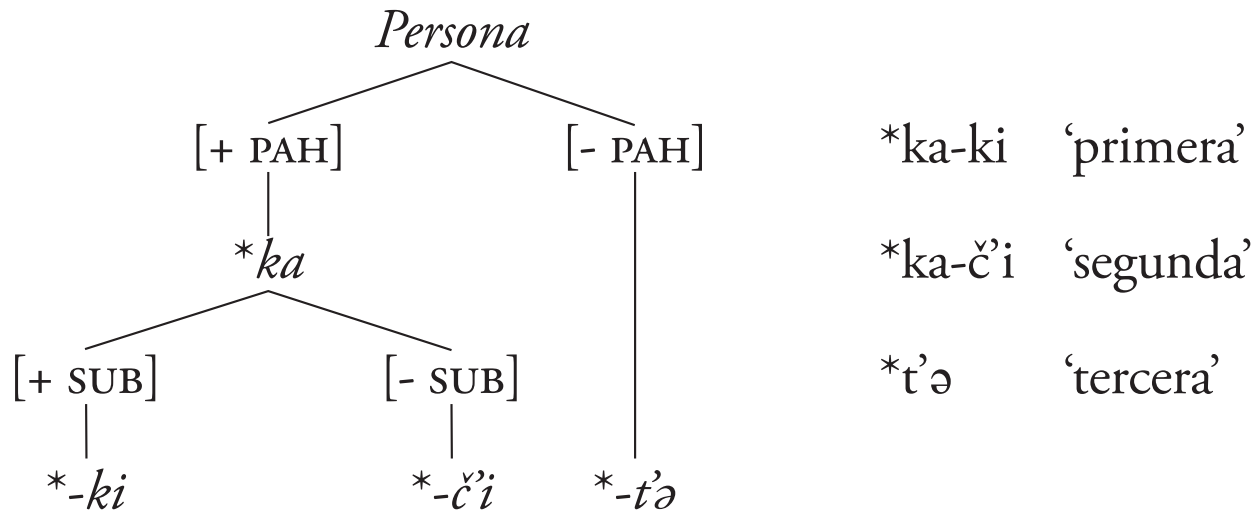


Por otro lado, en la categoría de número, la primera oposición se daba entre las formas plurales, con el rasgo [+PLURAL], y el singular, con el rasgo [-PLURAL]. Dentro de las formas plurales, se oponían el dual, con el rasgo [+DUAL], y el plural con el rasgo [-DUAL] (véase esquema 3). Las oposiciones de número con el rasgo [+PLURAL] se expresaban con afijos, mientras que el singular con el rasgo [-PLURAL] no tenía un exponente con estructura segmental, es decir, se mantenía la posición estructural dentro de la palabra para la categoría de número, pero no se llenaba. De esta manera,

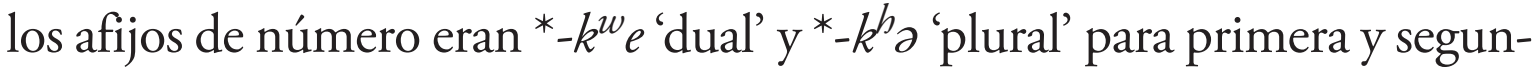
da persona, $\mathrm{y}^{*}$ - $h$ ' 'plural' para la tercera persona.

Esquema 3. Exponentes para las oposiciones dentro de la categoría de número

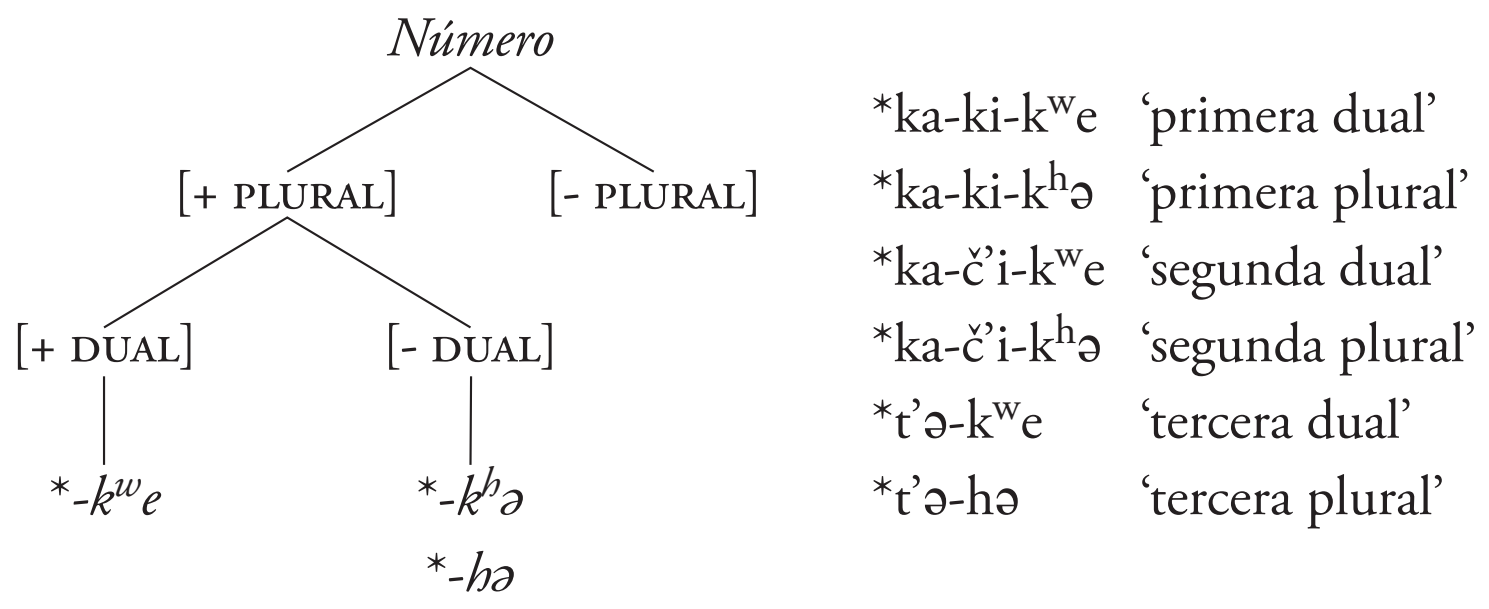

Finalmente, dentro de la primera persona [+PLURAL] se presentaba la oposición de exclusividad, expresando la diferencia entre exclusi$v o([+\mathrm{EXCL}])$ e inclusivo ([-EXCL]). En el protoatzinca, el término marcado de la oposición tanto en el ámbito de las categorías como en la expresión 
formal era el [+EXCLusivo], es decir, la exclusión de la segunda persona. Formalmente, los PPI en primera persona dual o plural exclusivos se marcaban con el sufijo - $b i$, pospuesto al afijo de número, mientras que las formas inclusivas no se marcaban con afijos (esquema 4).

Esquema 4. Oposiciones dentro de la categoría de exclusividad

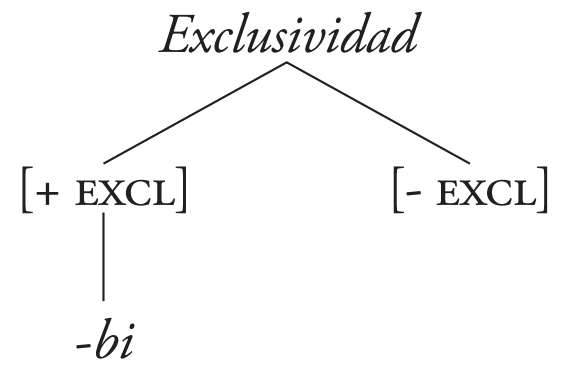

*ka-ki-kwe-bi 'primera dual exclusivo'

*ka-ki-k ${ }^{\mathrm{h}}$ ə-bi 'primera plural exclusivo'

El esquema 5 representa la jerarquía de las categorías gramaticales involucradas en el paradigma de los PPI del protoatzinca y que también funciona para las lenguas hijas, ya que, como mencioné, no hay cambios en las categorías expresadas y sus oposiciones. 
Esquema 5. Jerarquía de las categorías pronominales (CP) en los PPI del protoatzinca

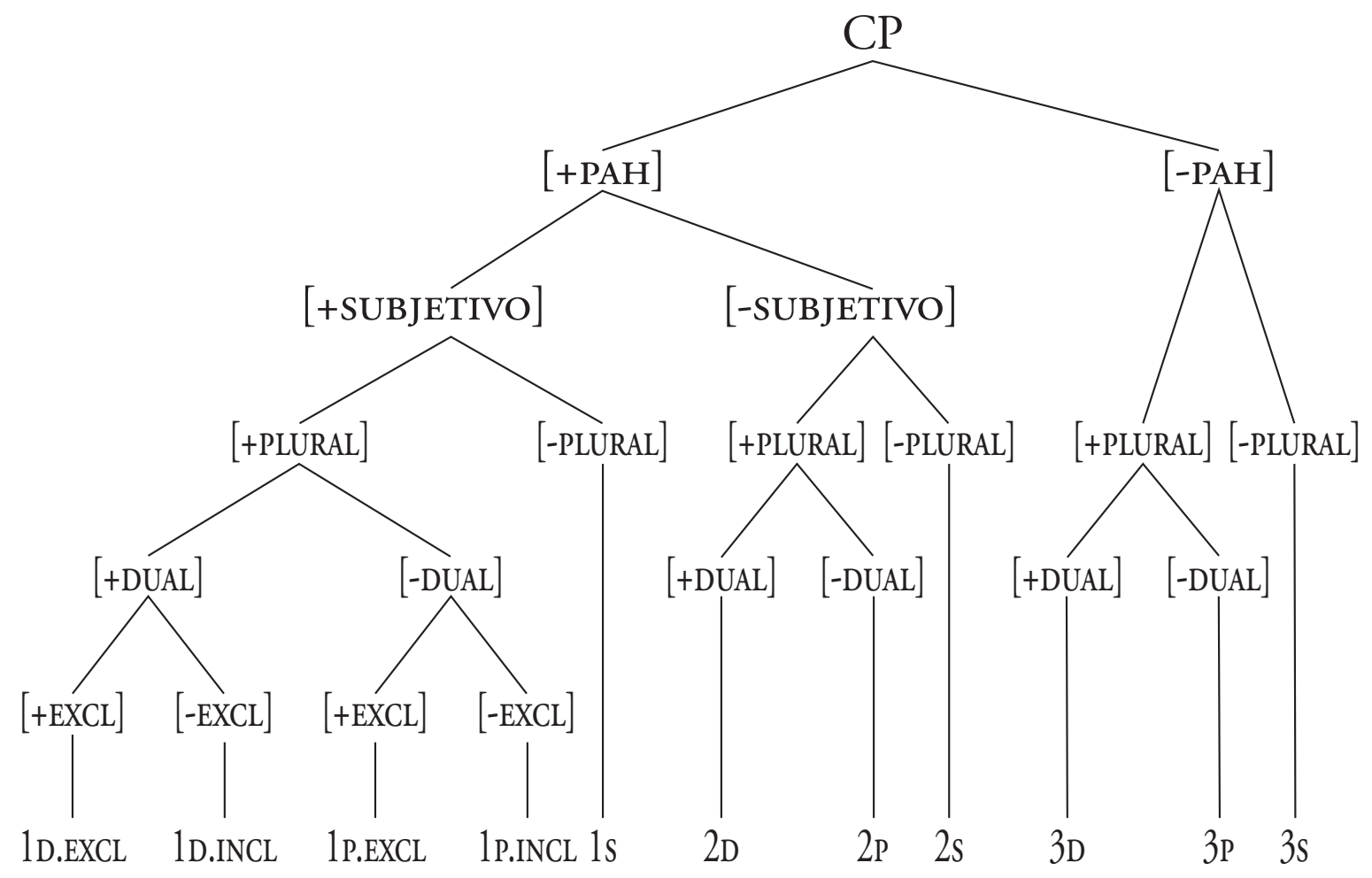

Como se verá en los siguientes apartados, esta jerarquía se refleja en el proceso de construcción de palabra del protoatzinca y las lenguas hijas.

\section{RECONSTRUCCIÓN DE LOS PPI DE PRIMERA Y SEGUNDA PERSONA}

En este apartado comienzo por presentar las formas cognadas de los PPI de primera y segunda personas y a continuación ofrezco la reconstrucción de la estructura de palabra morfológica y, finalmente, la reconstrucción fonológica de los exponentes morfológicos. En las tablas 1 y 2 se pueden observar las listas de cognados que comparé. Cada tabla está 
organizada por lenguas, número y exclusividad, en las formas de primera persona. En la mayoría de los casos hay más de una forma cognada, lo cual se debe a que hay formas distintas registradas por los autores. Las formas están escritas fonéticamente y los registros originales se pueden observar en el Anexo "Lista de cognados por lengua y fuente".

Tabla 1. PPI de primera persona en las lenguas atzincas

\begin{tabular}{|c|c|c|c|c|c|}
\hline $\begin{array}{l}\text { Lengua por } \\
\text { lugar }\end{array}$ & 1 singular & 1 dual & $\begin{array}{c}1 \text { dual } \\
\text { exclusivo }\end{array}$ & 1 plural & $\begin{array}{c}1 \text { plural } \\
\text { exclusivo }\end{array}$ \\
\hline Valle de Toluca & [kaki] & & & & [nkak $\left.{ }^{\mathrm{h}} \partial \mathrm{bi}\right]$ \\
\hline Undameo & [kaki] & {$\left[\mathrm{kak}^{\mathrm{w}}\right.$ ewi $]$} & $\begin{array}{l}{\left[\mathrm{kak}^{\mathrm{W}} \mathrm{ebi}\right]} \\
{\left[\mathrm{kak}^{\mathrm{W}} \mathrm{e} \text { epi }\right]}\end{array}$ & [kak $\left.{ }^{\mathrm{h}_{\text {owi }}}\right]$ & $\begin{array}{l}{\left[\mathrm{kak}^{\mathrm{h}} \partial \mathrm{Pbi}\right]} \\
{\left[\mathrm{kak}^{\mathrm{h}} \partial \mathrm{bi}\right]}\end{array}$ \\
\hline Charo & $\begin{array}{l}{[\mathrm{kaki}]} \\
{[\mathrm{kaaki}]}\end{array}$ & [kak ${ }^{\mathrm{w}}$ ewi] & {$\left[\mathrm{kak}^{\mathrm{w}} \mathrm{ebi}\right]$} & & {$\left[\mathrm{kak}^{\mathrm{h}} \partial \mathrm{bi}\right]$} \\
\hline Mexicaltzingo & $\begin{array}{l}\text { [gaki] } \\
\text { [kaki] }\end{array}$ & & & [kak ${ }^{\mathrm{h}}$ owi] & \\
\hline Matlatzinca* & [kaki] & & & [kak ${ }^{\mathrm{h}}$ owi] & \\
\hline $\begin{array}{l}\text { San Francisco } \\
\text { Oxtotilpan }\end{array}$ & $\begin{array}{l}{[\text { kaki] }} \\
\text { [kaki] }\end{array}$ & $\begin{array}{l}{\left[\mathrm{kak}^{\mathrm{w}} \text { ewi }\right]} \\
{\left[\mathrm{kák}^{\mathrm{w}} \text { ewi }\right]}\end{array}$ & $\begin{array}{l}{\left[\mathrm{kak}^{\mathrm{w}} \mathrm{ebi}\right]} \\
{\left[\mathrm{kaak}^{\mathrm{w}} \mathrm{e} \mathrm{ebi}\right]}\end{array}$ & $\begin{array}{l}{\left[\mathrm{kak}^{\mathrm{h}} \text { owi }\right]} \\
{\left[\mathrm{kak}^{\mathrm{h}} \text { ə?wi] }\right.} \\
{\left[\mathrm{kaak}^{\mathrm{h}} \text { əəwi] }\right.} \\
{\left[\mathrm{kak}^{\mathrm{h}} \mathrm{o}\right]}\end{array}$ & [kaak $\left.{ }^{\mathrm{h}} \partial \mathrm{Pbi}\right]$ \\
\hline San Juan Atzingo & 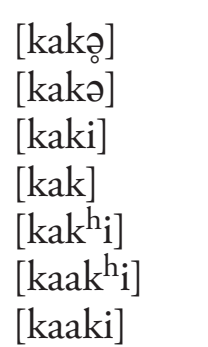 & $\begin{array}{l}{\left[\mathrm{kak}^{\mathrm{w}} \mathrm{e}\right]} \\
{\left[\mathrm{kak}^{\mathrm{h}} \mathrm{e}\right]}\end{array}$ & $\begin{array}{l}{\left[\mathrm{kak}^{\mathrm{w}} \mathrm{ebi}\right]} \\
{\left[\mathrm{kak}^{\mathrm{w}} \mathrm{ebi}\right]} \\
{\left[\mathrm{kak}^{\mathrm{wh}} \mathrm{ebi}\right]}\end{array}$ & {$\left[\mathrm{kak}^{\mathrm{h}} \mathrm{O}\right]$} & $\begin{array}{l}{\left[\mathrm{kak}^{\mathrm{h}} ə \mathrm{bi}\right]} \\
{\left[\mathrm{kák}^{\mathrm{h}} ə \mathrm{bi}\right]} \\
{\left[\mathrm{kak}^{\mathrm{h}} \mathrm{obi}\right]}\end{array}$ \\
\hline
\end{tabular}

* Utilizo la etiqueta "matlatzinca" en las tablas de formas cognadas, cuando los autores consultados no señalan si tomaron los datos de San Francisco Oxtotilpan o de Mexicaltzingo. 
Tabla 2. PPI de segunda persona en las lenguas atzincas

\begin{tabular}{|c|c|c|c|}
\hline Lengua por lugar & 2 singular & 2 dual & 2 plural \\
\hline Undameo & [kač́i] [kači $]$ & & $\begin{array}{l}\text { [kačowi] [kačowi] } \\
\text { [kačo] }\end{array}$ \\
\hline Charo & {$[$ kaači $]$} & [kačewi] & [kačowi] \\
\hline Mexicaltzingo & $\begin{array}{l}{[\mathrm{kači}][\mathrm{kaacci}]} \\
{[\text { kaač́i i] }}\end{array}$ & & [kačowi] \\
\hline Matlatzinca & [kači] [kaači i] & & [kačowi] \\
\hline San Francisco Oxtotilpan & 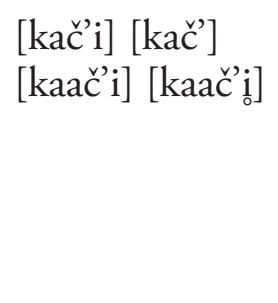 & $\begin{array}{l}\text { [kač'ewi] } \\
\text { [ka'č' ewi] } \\
\text { [kaač'ewi] } \\
\text { [kahčcewi] } \\
\text { [kahč'twí] } \\
\text { [kaahč' ewi] }\end{array}$ & $\begin{array}{l}{[\text { kahčcowi }]} \\
\text { [kahčcóowi }] \\
\text { [ka'čowi] }[\text { kač owi }] \\
{[\text { kačowi }]}\end{array}$ \\
\hline San Juan Atzingo & 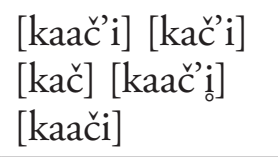 & 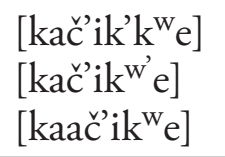 & $\begin{array}{l}\text { [kač'o] [kač'ik'o] } \\
{[\text { kačikho }} \\
{[\text { kaač́ikho] }}\end{array}$ \\
\hline
\end{tabular}

En las dos tablas se puede notar que hay diferencias entre los cognados. Dichas diferencias se deben, sin precisar por el momento, a tres factores: a) el registro de los distintos autores, b) diferencias por lenguas en la forma fonológica de algunos exponentes y reglas fonotácticas distintas, y c) variación sincrónica en cada lengua. Es importante señalar que la comparación y reconstrucción se hace a partir de las formas subyacentes de los pronombres, no de las formas fonéticas. En el siguiente apartado presento la reconstrucción y descripción de la estructura de palabra morfológica de los PPI en el protoatzinca. Posteriormente, presento la reconstrucción de los exponentes morfológicos con su forma fonológica subyacente. En este punto iré describiendo algunas de las diferencias entre las lenguas atzincas derivadas. 
3.1 Estructura de palabra morfológica de los PPI de primera y segunda persona del protoatzinca

Como ya mencioné, en los PPI de primera y segunda persona había una raíz pronominal y las oposiciones dentro de las categorías de persona, número y exclusividad se marcaban con un exponente morfológico afijal distinto para cada una de ellas. Así, los PPI de primera persona dual y plural eran palabras tetramorfémicas contando los exponentes para la oposición de exclusividad, mientras que el resto eran palabras trimorfémicas. En el esquema 6 muestro la jerarquización de la estructura de palabra morfológica de los PPI de primera y segunda personas del protoatzinca. Dicha estructura se mantiene sin cambios en las lenguas derivadas.

Esquema 6. Estructura de palabra morfológica de los PPI de primera y segunda persona del protoatzinca

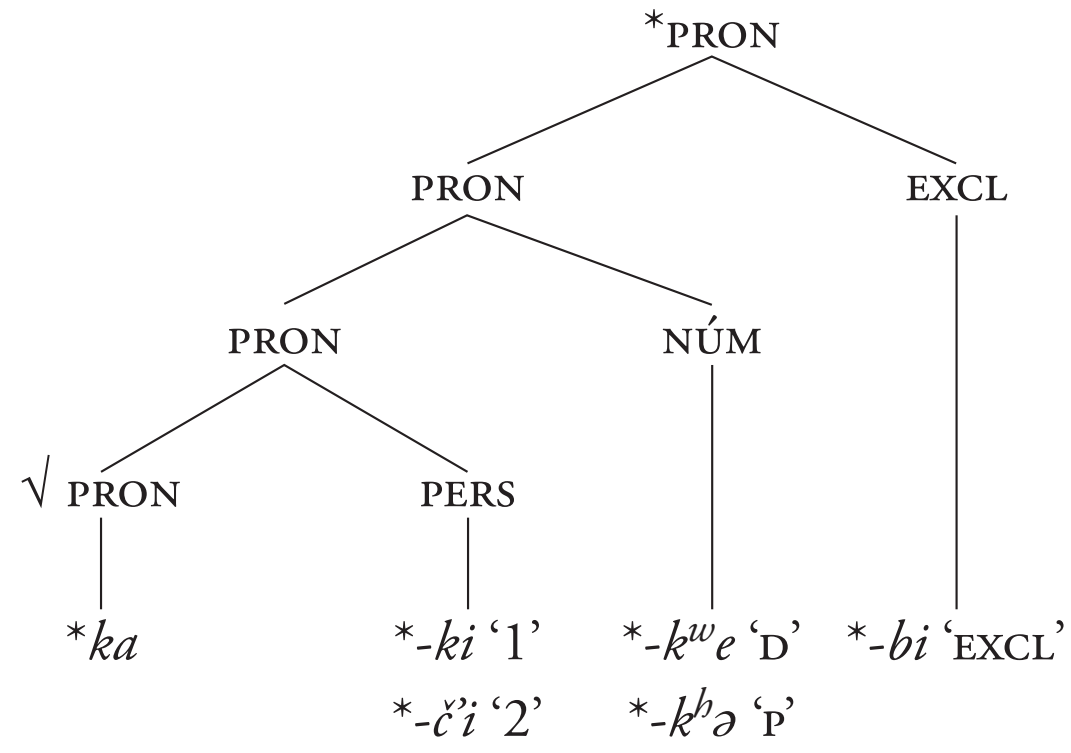


En el esquema 6 podemos observar que la posición de núcleo de la estructura estaba ocupada por la raíz pronominal para primera y segunda persona * $k a$. A la raíz se le sufijaban los exponentes de persona: ${ }^{*}-k i$ "primera persona" y ${ }^{*}-\check{c} i$ "segunda persona". Posteriormente se sufijaban los morfemas de número: ${ }^{*}-k^{w} e^{\text {'dual' }} \mathrm{y}^{*}-k^{h}$ a "plural”. Finalmente, se sufijaba el exponente de exclusividad ${ }^{*}-b i$ en los pronombres de primera persona dual y plural.

\section{Tabla 3. PPI del protoatzinca}

\begin{tabular}{|c|c|c|c|c|}
\hline & & Singular & Dual & Plural \\
\hline \multirow[t]{2}{*}{1} & Incl & ${ }^{*} \mathrm{ka}-\mathrm{ki}$ & ${ }^{*} \mathrm{ka}-\mathrm{ki}-\mathrm{k}^{\mathrm{w}} \mathrm{e}$ & ${ }^{*} \mathrm{ka}-\mathrm{ki}-\mathrm{k}^{\mathrm{h}} \mathrm{\partial}$ \\
\hline & Excl & & ${ }^{*} \mathrm{ka}-\mathrm{ki}-\mathrm{k}^{\mathrm{w}} \mathrm{e}-\mathrm{bi}$ & ${ }^{*} \mathrm{ka}-\mathrm{ki}-\mathrm{k}^{\mathrm{h}} \mathrm{\partial}-\mathrm{bi}$ \\
\hline 2 & & ${ }^{*} k a-c c^{\prime} i$ & ${ }^{*} k a-c^{\prime} i-k^{w} e$ & ${ }^{*} \mathrm{ka}-\mathrm{cr}^{\prime} \mathrm{i}-\mathrm{k}^{\mathrm{h}} \partial$ \\
\hline
\end{tabular}

En la tabla 3 presento las formas subyacentes de los PPI de primera y segunda del protoatzinca. Las formas que presento en el paradigma resultan de la reconstrucción de los morfemas sin pasar por las reglas y restricciones fonotácticas y silábicas, es decir, estas no necesariamente eran las formas superficiales de los PPI del protoatzinca. En la tabla 4 presento cada uno de los exponentes reconstruidos para los PPI de primera y segunda persona del protoatzinca, junto con la forma subyacente de los exponentes de los pronombres en los lenguas atzincas. 
Tabla 4. Forma fonológica de los morfemas de los PPI de las lenguas atzincas ${ }^{5}$

\begin{tabular}{|c|c|c|c|c|c|c|}
\hline & \multirow{2}{*}{$\begin{array}{c}\text { Raíz } \\
\text { Pronominal }\end{array}$} & \multicolumn{2}{|c|}{ Persona } & \multicolumn{2}{|c|}{ Número } & \multirow{2}{*}{$\begin{array}{c}\text { Exclusividad } \\
\text { Exclusivo }\end{array}$} \\
\hline & & Primera & Segunda & Dual & Plural & \\
\hline Protoatzinca & ${ }^{*} \mathrm{ka}$ & ${ }^{*}$-ki & $*_{\text {-Č́ } i}$ & ${ }^{*}-\mathrm{k}^{\mathrm{w}} \mathrm{e}$ & ${ }^{*}-\mathrm{k}^{\mathrm{h}} \partial$ & *-bi \\
\hline Toluca & $\mathrm{ka}$ & $-\mathrm{ki}$ & & & -həwi & -bi \\
\hline Undameo & $\mathrm{ka}$ & $-\mathrm{ki}$ & $-c^{\prime \prime} i$ & -wewi & -həwi & -bi \\
\hline Charo & $\mathrm{ka}$ & $-\mathrm{ki}$ & $-\check{c} \mathrm{i}$ & -wewi & -həwi & $-\mathrm{bi}$ \\
\hline Mexicaltzingo & $\mathrm{ka}$ & $-\mathrm{ki}$ & $-c^{\prime \prime} i$ & -wewi & -həwi & $-b i$ \\
\hline San Francisco Oxtotilpan & $\mathrm{ka}$ & $-\mathrm{ki}$ & $-c^{\prime} \mathrm{i}$ & -wewi & -həwi & -bi \\
\hline San Juan Atzingo & $\mathrm{ka}$ & $-\mathrm{ki}$ & $-c^{\prime} \dot{i}$ & $-\mathrm{k}^{\mathrm{w}} \mathrm{e}$ & $-\mathrm{k}^{\mathrm{h}} \partial$ & -bi \\
\hline
\end{tabular}

En la tabla 4 se puede observar que los cambios en la estructura fonológica subyacente de los morfemas que componen a los PPI de primera y segunda persona se encuentra en los sufijos de número. En el protoatzinca los exponentes de número eran ${ }^{*}-k^{w} e$ 'dual' $y^{*}-k^{h} \partial$ 'plural'. La forma fonológica de estos sufijos se mantiene en la lengua de San Juan Atzingo, mientras que en el resto de las lenguas atzincas ambos sufijos perdieron el segmento * $[\mathrm{k}]$ de la consonante inicial, y se fonologizó la secuencia /wi/ como parte de su estructura, resultando /-wewi/ y /-howi/ respectivamente. Por otro lado, la raíz pronominal y los exponentes morfológicos para las categorías de persona y de exclusividad, se mantienen sin cambios subyacentes del protoatzinca hacia las lenguas derivadas.

5 En esta y las siguientes tablas ya no incluyo la lengua etiquetada como "matlatzinca" en las tablas de formas cognadas anteriores, en tanto que se trata de las lenguas de San Francisco Oxtotilpan o Mexicaltzingo. 


\subsection{Reconstrucción fonológica de los exponentes morfológicos ${ }^{6}$}

La raíz pronominal *ka era el núcleo de la estructura de palabra de los PPI con el rasgo [+PAH], es decir, los de primera y segunda personas. Se trataba de una raíz por posición estructural, ya que era el núcleo de la palabra. La raíz estaba formada por la consonante densa grave interrupta */k/ y la vocal densa */a/. En la lengua de Mexicaltzingo la consonante está registrada como sorda $[\mathrm{k}]$ o sonora $[\mathrm{g}]$. No tengo elementos para explicar el contexto de sonorización, ya que el pronombre está registrado sin elementos que le antecedan. Carranza (2011) describe este mismo cambio en los PPI de la lengua de San Francisco Oxtotilpan, en especial en el habla continua en las generaciones mayores. En esta lengua la consonante sonora [g] es alófono del fonema denso grave interrupto $/ \mathrm{k} / .^{7}$ Sin embargo, dicho fonema en inicio de palabra tiende a realizarse sordo $[\mathrm{k}]$. El templete de la raíz era ${ }^{*} \mathrm{CV}$ con vocal monomoraica.

En la tabla 5 presento las realizaciones de la raíz en cada lengua. Podemos notar que en la lengua de Undameo la raíz se realiza siempre con vocal corta, en la de Charo la primera persona singular presenta variación entre vocal corta y larga, la segunda persona tiene vocal larga y en el resto de los pronombres hay vocal corta. Considero que no hay suficientes datos para las lenguas de Toluca y Mexicaltzingo para encontrar una regularidad o tendencia; solo hay variación en el de segunda persona singular de Mexicaltzingo. En la lengua de San Francisco Oxtotilpan, así como en

${ }^{6}$ Para la descripción fonológica utilizo los rasgos propuestos por Trubetzkoy (1973[1939]) y Jakobson y Waugh (1979).

7 Véase Pascacio (2006). 
la de San Juan Atzingo, la tendencia es que la vocal de la raíz sea larga en los PPI de segunda y corta en los de primera.

Tabla 5. Realización de la raíz pronominal en las lenguas atzincas

\begin{tabular}{|c|c|c|c|c|c|c|}
\hline & Toluca & Undameo & Charo & Mexicaltzingo & $\begin{array}{c}\text { San Francisco } \\
\text { Oxtotilpan }\end{array}$ & $\begin{array}{l}\text { San Juan } \\
\text { Atzingo }\end{array}$ \\
\hline $1 \mathrm{~s}$ & {$[\mathrm{ka}]$} & {$[\mathrm{ka}]$} & {$[\mathrm{ka}]-[\mathrm{kaa}]$} & {$[\mathrm{ka}] \sim[\mathrm{ga}]$} & {$[\mathrm{ka}]$} & {$[\mathrm{ka}]-[\mathrm{kaa}]$} \\
\hline $1 \mathrm{D}$ & & {$[\mathrm{ka}]$} & {$[\mathrm{ka}]$} & & {$[\mathrm{ka}]$} & {$[\mathrm{ka}]$} \\
\hline $1 \mathrm{DE}$ & & [ka] & {$[\mathrm{ka}]$} & & {$[\mathrm{ka}]-[\mathrm{kaa}]$} & {$[\mathrm{ka}]$} \\
\hline 1P & & [ka] & & {$[\mathrm{ka}]$} & {$[\mathrm{ka}]$} & {$[\mathrm{ka}]$} \\
\hline $1 \mathrm{PE}$ & [ka] & [ka] & {$[\mathrm{ka}]$} & & [kaa] & {$[\mathrm{ka}]$} \\
\hline $2 s$ & & {$[\mathrm{ka}]$} & [kaa] & {$[\mathrm{ka}]-[\mathrm{kaa}]$} & {$[\mathrm{ka}]-[\mathrm{kaa}]$} & {$[\mathrm{ka}]-[\mathrm{kaa}]$} \\
\hline $2 \mathrm{D}$ & & {$[\mathrm{ka}]$} & {$[\mathrm{ka}]$} & & $\begin{array}{l}{[\mathrm{ka}] \sim[\mathrm{kaa}] \sim[\mathrm{kah}] \sim} \\
{[\mathrm{kaah}]}\end{array}$ & {$[\mathrm{ka}]-[\mathrm{kaa}]$} \\
\hline $2 \mathrm{P}$ & & {$[\mathrm{ka}]$} & {$[\mathrm{ka}]$} & {$[\mathrm{ka}]$} & {$[\mathrm{ka}]-[\mathrm{kah}]$} & {$[\mathrm{ka}]-[\mathrm{kaa}]$} \\
\hline
\end{tabular}

Reconstruyo la raíz de los PPI del protoatzinca con el templete *CV con vocal monomoraica, pero no hay argumentos contundentes para reconstruir la vocal como monomoraica o bimoraica. Presento, en relación con esto, tres puntos a considerar: a) en las lenguas coloniales de Undameo y Charo la raíz está registrada en la mayoría de los casos con la vocal corta, mientras que en las lenguas actuales de San Francisco Oxtotilpan y San Juan Atzingo, la tendencia es que en los pronombres de primera persona la raíz se realice con vocal corta, mientras que en los de segunda persona se realiza con vocal larga; b) en las lenguas atzincas las vocales bimoraicas solo se presentan en las raíces léxicas, por lo tanto la vocal bimoraica en la raíz pronominal es posible; y c) Bartholomew (1965:217), al explicar el 
desarrollo desde el proto-otopame al matlatzinca y ocuilteco, señala que las formas matlatzincas para los pronombres independientes antepusieron $k a$-, con vocal corta, a los morfemas de persona. Para continuar con la argumentación sobre la moraicidad de la vocal de la raíz, es necesario rastrear el origen de la raíz pronominal y tomar en cuenta los cambios diacrónicos descritos por Bartholomew $(1965,1992)$ respecto de la cantidad moraica de las raíces léxicas.

Los exponentes morfológicos para la primera y segunda personas eran

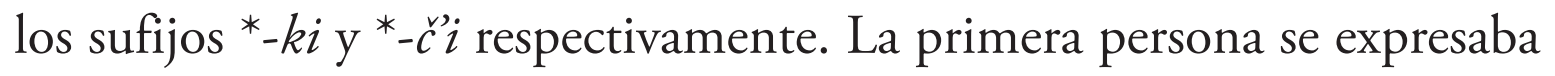
con el sufijo formado por la consonante densa grave interrupta */k/ y la vocal aguda difusa $* / \mathrm{i} /$, mientras que la segunda persona se expresaba con el sufijo formado por la consonante densa, aguda, interrupta, glotalizada */č'/ y la vocal aguda difusa */i/. El templete de estos afijos era ${ }^{*} \mathrm{CV}$, mismo que encontramos en las lenguas atzincas derivadas para los afijos. En la lengua de Charo no hay registro de la glotalización del sufijo de segunda persona. Sin embargo, considero que esto se debe a que el registro de Basalenque (1975a,b [1642]) del sonido glotal [?] no fue regular, ni cuando se trataba de la consonante (/P/), ni cuando era rasgo consonántico (C') o particularidad prosódica (V’, V’V) (Trubetzkoy 1973[1939]). Para la lengua de Mexicaltzingo, tomo como registro de la glotalización del sufijo de segunda persona, el registro de Hasler (1961) del pronombre de segunda persona singular [kaači i]. En la lengua de San Juan Atzingo solamente un autor no registra la glotalización de la consonante del sufijo de segunda persona, pero los otros cognados sí la marcan. Así pues, considero que en los exponentes para la categoría de persona no hay cambios del protoatzinca a las lenguas derivadas. 
En los pronombres de primera persona singular de la lengua de San Juan Atzingo, la vocal /i/ con los rasgos [+aguda] [+difusa] se realiza como [ə] con los rasgos [-densa] [-difusa] [+bemolizada]. Este cambio puede estar relacionado con la consonante $/ \mathrm{k} /$ del sufijo de primera persona, ya que tiene los rasgos [+denso] [+grave] y este no ocurre con la consonante de la segunda persona /č'/ con el rasgo [+agudo]. Otro cambio sincrónico relacionado con la vocal de los exponentes de persona es que se elide ante los sufijos de número. Este cambio se presenta en los pronombres de todos las lenguas atzincas, exceptuando los de segunda persona de la lengua de San Juan Atzingo, en los cuales se mantiene la vocal.

Los exponentes para las oposiciones de la categoría de número eran ${ }^{*}-k^{w} e$ 'dual' $\mathrm{y}^{*}-k^{h}$ a "plural", considerando que el singular no se expresaba, aunque sí se mantenía la posición estructural en la palabra morfológica. Ambos sufijos tenían el templete $\mathrm{CV}$, el dual estaba formado por la consonante densa grave interrupta bemolizada $* / \mathrm{k}^{\mathrm{w}} /$ y la vocal aguda */e/, mientras que el plural estaba formado por la consonante densa grave interrupta tensa $* / \mathrm{k}^{\mathrm{h}} /$ y la vocal bemolizada $* /$ ə $/$.

Estos afijos sí presentan cambios del protoatzinca hacia las lenguas derivadas. La lengua de San Juan Atzingo mantiene la forma fonológica de los exponentes del protoatzinca, mientras que en el resto de las lenguas hubo dos cambios. Uno de ellos consiste en que estos morfemas perdieron la consonante obstruyente velar $[\mathrm{k}]$ al inicio, conservando la bemolización o aspiración como consonantes plenas y no como rasgos secundarios. El otro cambio es que estos afijos incorporaron la secuencia final /wi/ a su forma fonológica, resultando -howi "plural" y -wewi “dual". En el esquema 7 se pueden observar los cambios en cada exponente. Estos cambios son 
importantes en tanto que ambos separan a la lengua de San Juan Atzingo del resto de las lenguas atzincas.

Esquema 7. Cambios en los exponentes de número
(a) $* /-\mathrm{k}^{\mathrm{w} e} /$ "dual" $>/-\mathrm{k}^{\mathrm{w} e} /$
San Juan Atzingo
$>$ /-wewi/
El resto de las lenguas atzincas
(b) $* /-\mathrm{k}^{\mathrm{h}} \mathrm{\rho} /$ "plural" $>/-\mathrm{k}^{\mathrm{h}} \mathrm{\partial} /$
San Juan Atzingo
$>$ /-həwi/
El resto de las lenguas atzincas

Es posible reconstruir los exponentes de número dual y plural con el segmento consonántico ${ }^{*}[\mathrm{k}]$ gracias a los pronombres de segunda persona dual y plural de la lengua de San Juan Atzingo. En estos pronombres se mantiene la vocal del sufijo de segunda persona $-c ̌ c i$ al sufijarse los morfemas de número, de manera que no hay restricciones fonotácticas y se mantiene sin cambios el segmento inicial de los sufijos de número. En los pronombres de primera persona de la lengua de San Juan Atzingo, sí se elide la vocal difusa aguda /i/ del sufijo de persona $-k i$; el resultado es la secuencia de la consonante del sufijo de primera persona $/ \mathrm{k} /$ seguida de la consonante inicial de los sufijos de número $/ \mathrm{k}^{\mathrm{w}} / \mathrm{y} / \mathrm{k}^{\mathrm{h}} /$. Estas secuencias $\left(/ \mathrm{kk}^{\mathrm{w}} /, / \mathrm{kk}^{\mathrm{h}} /\right)$ no están permitidas y, por lo tanto, uno de los segmentos idénticos se elide, resultando una sola consonante densa grave interrupta ya sea bemolizada $/ \mathrm{k}^{\mathrm{w}} /$ en el caso del dual, o tensa $/ \mathrm{k}^{\mathrm{h}} /$ en el caso del plural.

En el resto de las lenguas atzincas, tanto en los pronombres de primera persona como en los de segunda, se elide la vocal difusa aguda /i/ de los sufijos de persona -kiy - $c^{r} i$. Debido a la elisión, quedan secuencias no permitidas de dos consonantes (/kh/, /kw/, /čh/, /čww/). En el caso de los PPI 
de primera, la consonante inicial de los exponentes de número $(/ \mathrm{h} /, / \mathrm{w} /)$ se mantiene como rasgo de la consonante del morfema de primera persona, resultando las consonantes $/ \mathrm{k}^{\mathrm{w}} / \mathrm{y} / \mathrm{k}^{\mathrm{h}} /$ respectivamente, mientras que en los pronombres personales de segunda persona se elide la consonante inicial de los sufijos de número.

Por otro lado, tenemos la fonologización de la secuencia /wi/ como parte de los sufijos de número -wewi 'dual' y -howi 'plural'. La secuencia /wi/ es una sílaba final regular de las palabras nominales y verbales de las lenguas atzincas exceptuando la de San Juan Atzingo. Bartholomew (1965) propone que el matlatzinca (agrupando la lengua de San Francisco Oxtotilpan y coloniales) inserta el sufijo -wi después de la sílaba de una raíz y antes de la frontera de palabra, por ejemplo cuando no hay un sufijo formativo de radical. ${ }^{8}$ En términos de templete, esto se puede interpretar como que el matlatzinca estaría agregando la secuencia /wi/ a las raíces con templete CV en final de palabra generando el templete CVwi. Muntzel (1986:78) sugiere que la lengua de San Juan Atzingo perdió dicho sufijo, lo cual implica que lo tenía, al igual que el matlatzinca. Sin embargo, no tengo elementos que me permitan saber si la lengua de San Juan Atzingo lo incorporó y después lo perdió, o si nunca lo incorporó. Hasta el momento, ningún autor le ha atribuido una función morfológica o significado gramatical a la secuencia /wi/, ni en las lenguas coloniales, ni en las actuales.

En las lenguas atzincas, exceptuando a San Juan Atzingo, los sufijos de número dual y plural en palabras nominales son -wewi y -ha. La hipóte-

8 Según Bartholomew (1965:197), "Matlatzinca inserts a suffix -wi after a root syllable and before word boundary, i.e., when there is no stem-formative suffix." 
sis que propongo para las lenguas que incorporaron la secuencia/wi/ a los morfemas de número es que en el sufijo de dual en nominales y posteriormente en los PPI de tercera persona se reanalizó la secuencia /wi/ como parte de su estructura fonológica subyacente -wewi, esto probablemente por analogía con la regularidad de la secuencia final /wi/ tanto en palabras nominales como verbales. Posteriormente, en los PPI de primera y segunda persona también se agregó y reanalizó la secuencia /wi/ como parte de la estructura fonológica, no solo del morfema de dual, sino también del de plural para regularizar el templete fonológico de palabra, resultando -wewi y -hawi. Sin embargo, en los pronombres de tercera persona plural y en las palabras nominales no se incorporó la silaba final /wi/ como parte del sufijo de plural, quedando como - $h$.

En las tablas 5 y 6 podemos observar, en los PPI en plural, que la primera vocal del sufijo de número presenta una alternancia realizándose como aguda bemolizada [ə] o como grave [o] ([həwi] $\sim$ [howi $] \mathrm{o}\left[\mathrm{k}^{\mathrm{h}} \mathrm{\partial}\right] \sim\left[\mathrm{k}^{\mathrm{h}} \mathrm{o}\right]$. La tendencia es que la vocal bemolizada [ə] aparece generalmente en los pronombres de primera persona exclusivos, mientras que la vocal grave [o] aparece generalmente en los pronombres inclusivos, así como en los pronombres de segunda persona. Por otro lado, en los PPI de las lenguas que incorporaron la silaba final /wi/ como parte de las marcas de número también podemos observar que en los de primera dual y plural la silaba /wi/ de los sufijos de número pierde la vocal difusa aguda /i/ ante el sufijo de exclusividad -bi. En este caso, la consonante /w/ se realiza como glotal [?]. De estos cambios resultan formas como [kaakwe?bi] o [kaak ${ }^{\mathrm{h}} \partial \mathrm{Pbi}$. En la tabla 6 de cognados se puede observar la forma [kačo] para el pronombre de segunda persona plural de la lengua de San Juan Atzingo, registra- 
da por Soustelle (1993 [1937]). Esta forma queda fuera de la regularidad que se puede observar en los cognados y parece corresponder más bien, a otra de las lenguas atzincas, ya sea de Mexicaltzingo o de San Francisco Oxtotilpan.

También llaman la atención las formas [kači $i k^{\prime} k^{w} e$ ], [kač $\left.i k^{w^{\prime}} e\right]$ y [kač'ik'o]. Schumann (1975) registra el primer caso para la lengua de San Juan Atzingo. Se puede observar una consonante interrupta densa grave glotalizada /k'/ que no está registrada por ningún otro autor en esa posición. Silábicamente, solo puede ser la coda de la segunda sílaba [ka.či ik’. $\mathrm{k}^{\mathrm{w}} \mathrm{e}$ ] o la primera consonante del inicio ramificado de la tercera sílaba de izquierda a derecha [ka.či. $k^{\prime} k^{\mathrm{w}} \mathrm{e}$ ], pero ninguna de las dos posiciones está permitida para esta consonante en la lengua de San Juan Atzingo. Por otro lado, tenemos las formas [kač' $\mathrm{ik}^{\mathrm{w}} \mathrm{e}$ ] y [kačik'o] registradas por Muntzel (1986) también para San Juan Atzingo. En estos registros, la consonante de los sufijos de número dual y plural está glotalizada $/ \mathrm{k}^{\mathrm{w}} / \mathrm{y} / \mathrm{k} /$. En el resto de los registros la consonante del sufijo de número dual no está glotalizada y la de número plural es tensa no glotalizada. No considero que estas diferencias se deban a los registros. Sin embargo, por el momento no tengo suficientes datos que me permitan explicar la variación entre dichas consonantes glotalizadas y no glotalizadas.

Por último, también observamos la forma [kahčłiwi] de la lengua de San Francisco Oxtotilpan, registrada por Escalante y Hernández (1994). En este caso, la vocal del sufijo de dual se realiza como bemolizada y difusa [i]. Aunque la bemolización se podría explicar por la consonante bemolizada /w/, esta alternancia no la encontramos registrada por ningún otro autor, ni por los mismos Escalante y Hernández (1999a, s.f. a y s.f. b), así que no cuento con datos para saber si realmente se presenta. 
El sufijo de exclusividad se mantiene sin cambios del protoatzinca hacia las lenguas derivadas. Este morfema tenía el templete CV que también se encuentra para los afijos en las lenguas derivadas. Estaba formado por la consonante difusa grave continua */b/ y la vocal aguda difusa */i/. Es necesario mencionar que en las fuentes coloniales no se señala la oposición exclusivo : inclusivo, aunque, a partir de la fonologización y la sistematización morfológica, se puede proponer que en las lenguas atzincas coloniales sí se oponían las formas exclusivas e inclusivas.

Una tendencia regular que se puede observar en las tablas 5 y 6 de cognados es que la vocal aguda difusa /i/ se ensordece en final de palabra. En la tabla 5 de cognados, se observa que en el segmento final de palabra de los pronombres de primera persona de la lengua de San Juan Atzingo hay mucha variación. Encontramos la vocal [i], la vocal [ə], esta misma vocal sorda [ə̊], la ausencia de vocal y la vocal [i] pero con la consonante del morfema tensa $\left[\mathrm{k}^{\mathrm{h}}\right]$. Esta variación está relacionada con el proceso de ensordecimiento y con el de pérdida de rasgos. En los pronombres exclusivos solo se encuentra la vocal [i] y esta misma vocal sorda [i] persona singular encontramos estas mismas vocales y la ausencia de vocal. En el resto de las lenguas encontramos la vocal [i] y esta misma vocal sor$\mathrm{da}$ [i]. Tanto el ensordecimiento de las vocales /i/ y /a/ en final de palabra, como la elisión de la vocal/i/ en los morfemas de persona y de número, probablemente están relacionados con posiciones átonas de la palabra.

\section{ReCONSTRUCCIÓN DE LOS PPI DE TERCERA PERSONA}

Los PPI de tercera persona tienen el rasgo [-PAH] en oposición a los de primera y segunda personas con el rasgo $[+\mathrm{PAH}]$. Como ya he mencionado, 
esto encuentra evidencia formal en que tienen un comportamiento morfológico distinto, ya que en los pronombres de tercera persona el núcleo de la estructura de palabra morfológica es el exponente de tercera persona. Cabe mencionar que los demostrativos pueden funcionar como pronombres personales de tercera persona, aunque para el análisis que realizo excluyo a los demostrativos. En la tabla 6 presento la lista de cognados de pronombres de tercera persona de las lenguas atzincas derivadas. El arreglo de las tablas se basa en lengua y la categoría de número.

Tabla 6. PPI de tercera persona de las lenguas atzincas

\begin{tabular}{|c|c|c|c|}
\hline Lengua por lugar & Singular & Dual & Plural \\
\hline Valle de Toluca & [kit'awi] & & \\
\hline Undameo & $\begin{array}{l}\text { [ret'ewi] [det'ewi] } \\
\text { [detewi] [kit'ewi] } \\
\text { [int'ewi] [intewi] }\end{array}$ & [int'ewewi] & 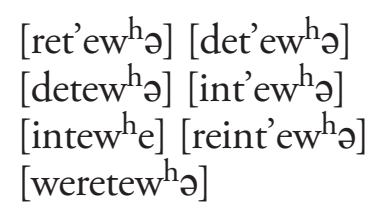 \\
\hline Charo & $\begin{array}{l}\text { [det'ewi] } \\
\text { [indegewi] } \\
\text { [kiregewi] } \\
\text { [int'ewi] }\end{array}$ & [int'ewewi] & [int'ew ${ }^{\mathrm{h}}$ ə] \\
\hline Matlatzinca & [reCəwi] [re't’’] & & {$\left[\right.$ retəw $\left.^{\mathrm{h}} \partial\right]$} \\
\hline San Francisco Oxtotilpan & $\begin{array}{l}\text { [ret'owi] [ret'ow i] } \\
\text { [ret'iwi] [reet'owi] } \\
\text { [t'owi] [t'tiwi] } \\
\text { [tat'iwi] [kat'ewi] }\end{array}$ & $\begin{array}{l}\text { [ret'owewi] } \\
\text { [reet'owewi] } \\
\text { [ret'iwewi] } \\
\text { [ret'ewewi] [ret'owə] } \\
\text { ['owewi] }\end{array}$ & 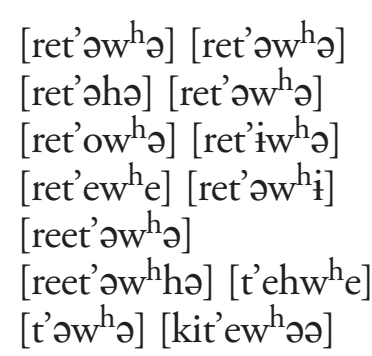 \\
\hline San Juan Atzingo & $\begin{array}{l}\text { [lyehtye] [lyete] } \\
\text { [let'o] [leht'yi] } \\
\text { [liht'ə] [leigt'e] } \\
\text { [léht'ə] }\end{array}$ & $\begin{array}{l}\text { [lyetəngwe] } \\
\text { [leht'yinwe] } \\
\text { [liht'ənkwe] } \\
\text { [lyaht' ingwe] }\end{array}$ & $\begin{array}{l}\text { [lyetenyə] [leht' yihñ̄a] } \\
\text { [liht’ohñ] [leigt'eñe] } \\
\text { [lyəht'inhyã] }\end{array}$ \\
\hline
\end{tabular}


En la tabla 6 podemos observar diferencias entre los pronombres de tercera persona que se deben al registro de los autores, a cambios en la estructura de palabra morfológica y a cambios en la función y forma fonológica de los exponentes. En contraste con los pronombres de primera y segunda personas, en los de tercera sí hay cambios diacrónicos estructurales en la palabra morfológica del protoatzinca hacia las lenguas atzincas derivadas. Es importante señalar que para la reconstrucción utilizo las formas cognadas que inician con las secuencias [re], [de], [lye], en tanto que son las más regulares y recurrentes para el pronombre de tercera persona en los datos con los que cuento.

\subsection{Estructura de palabra morfológica de los PPI de tercera del protoatzinca}

En el protoatzinca los pronombres de tercera persona independientes expresaban con morfemas las categorías de persona y número y tenían el prefijo ${ }^{*}$ re-. La categoría de persona expresaba la oposición [-PAH] con el exponente de tercera persona * t’̉ como núcleo. Las oposiciones de número dual y plural se expresaban con los exponentes sufijales * $k^{w} e{ }^{*}$ - ho respectivamente, mientras que el singular no tenía un exponente afijal. La posición más lejana a la raíz estaba ocupada por el prefijo * re-cuya categoría o función aún no está definida. En el esquema 8 de estructura de palabra morfológica utilizo la etiqueta $\mathrm{R}$ para señalar la posición estructural jerárquica de este prefijo sin pretender asociarla con una categoría específica. 
Esquema 8. Estructura de palabra morfológica de los PPI de tercera persona del protoatzinca

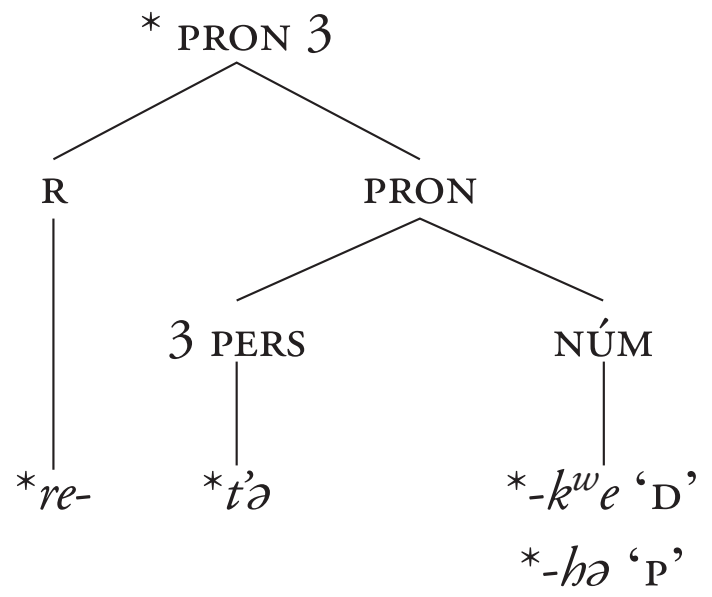

En la tabla 7 presento el paradigma de pronombres de tercera persona. $\mathrm{Al}$ igual que en el caso del paradigma de pronombres de primera y segunda persona, las formas que presento en la tabla resultan de la reconstrucción de los morfemas sin pasar por las reglas y restricciones fonotácticas y silábicas, es decir, estas no necesariamente eran las formas superficiales de los pronombres del protoatzinca.

Tabla 7. PPI de tercera persona del protoatzinca

\begin{tabular}{ccc}
\hline Singular & Dual & Plural \\
\hline$*^{*}$ re-t'ə & ${ }^{*}$ re-t'ə-k $\mathrm{k}^{\mathrm{w}} \mathrm{e}$ & ${ }^{*}$ re-t'ə-hə \\
\hline
\end{tabular}

La estructura de palabra de los pronombres independientes de tercera persona cambió en la lengua de San Juan Atzingo, mientras que se mantuvo igual en el resto de las lenguas. El cambio estructural en la lengua de San Juan Atzingo radica en que el prefijo ${ }^{*}$ re- se gramaticalizó como 
parte del exponente de tercera persona. Así, el núcleo de la estructura es la secuencia leht’ y a este exponente se le sufijan los exponentes de número. En el esquema 9 presento esta estructura de palabra morfológica.

Esquema 9. Estructura de palabra morfológica de los PPI de tercera persona de San Juan Atzingo

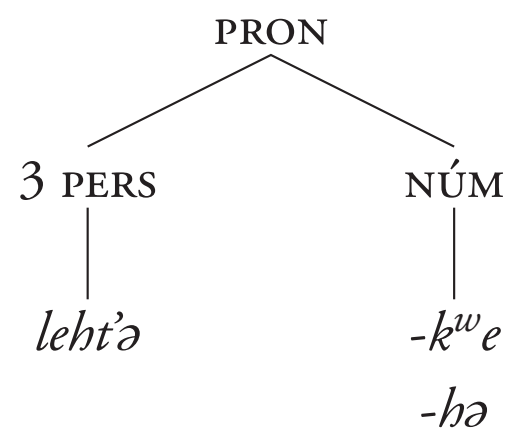

Propongo este cambio ya que no hay evidencia para segmentar morfológicamente la secuencia leht’. Muntzel (1986:90) también presenta esta secuencia como monomorfémica. El cambio de la consonante */r/ del protoatzinca a la consonante /1/ de la lengua de San Juan Atzingo, lo explico más adelante.

\subsection{Reconstrucción fonológica de los exponentes morfológicos}

El exponente ${ }^{*} \grave{\partial}$ expresaba tercera persona y era el núcleo morfológico del pronombre. Tenía el templete CV y estaba formado por la consonante aguda difusa interrupta glotalizada */t’ / y la vocal bemolizada /ə/. Como mencioné, en la lengua de San Juan Atzingo, la secuencia /leh/se gamaticalizó como parte del exponente resultando leht’’ (esquema 10). 
Esquema 10. Cambios en los PPI de tercera persona

*'̉े > leht’o Lengua de de San Juan Atzingo

$>$ towi El resto de las lenguas atzincas

En el resto de las lenguas la secuencia /wi/ se gramaticalizó como parte del exponente resultando t’awi (esquema 10). En contraste con el cambio en la lengua de San Juan Atzingo, la gramaticalización de /wi/ no afectó la estructura de palabra morfológica sino solo la forma fonológica del exponente de persona. Este cambio podría estar relacionado con la regularidad de la secuencia /wi/ al final de las palabras nominales y verbales.

En la tabla 6 de cognados podemos observar las formas [indegewi] y [kiregewi] registradas por Basalenque (1975a [1642]). El autor las presenta para describir la alternancia entre $[\mathrm{r}]$ y $[\mathrm{d}]$, indicando que se trata de la tercera persona y las escribe como <indegehuí> y <quiregehuí>. En estos casos, al parecer la consonante densa grave $[\mathrm{g}]$ corresponde a la consonante del exponente de persona, sin embargo no encuentro elementos, que permitan explicar la alternancia [ $\left.\mathrm{t}^{\prime}\right] \sim[\mathrm{g}]$ en su registro.

En las formas cognadas también podemos observar la forma [reCəwi], en este caso con $\mathrm{C}$ indico que en ese lugar debe haber una consonante. Este registro pertenece a Soustelle (1993[1937]), donde la forma original es <ręöwi $>$. En la secuencia no se observa la consonante del exponente de persona, sin embargo, indico la presencia de la consonante porque en las lenguas atzincas no se permite la secuencia de vocales diferentes y por los otros cognados presentados en la tabla 6. En la lengua de San Juan Atzingo, las consonantes agudas difusas tienden a volverse sostenidas. A esto se debe que Schumann (1975) y Soustelle (1993[1937]) hayan registrado las 
secuencias $[t y]$ y [ $\left.\mathrm{t}^{\prime} \mathrm{y}\right]$. Por último, Soustelle es el único autor que no registra la glotalización de esta consonante.

En cuanto a la calidad de la vocal del exponente, considero que en el protoatzinca el exponente de tercera persona tenía una vocal bemolizada ya fuera difusa */i/ o no */ə/. En la tabla 11, se puede observar que está registrada como vocal bemolizada difusa / $\mathbf{i} /$ y no difusa /ə/ en las lenguas derivadas. Aunque también se puede observar como /e/ ([-densa] [-difusa] [+aguda]), /a/ ([+densa]) y /o/ ([-densa] [-difusa] [+grave]). La vocal /a/ está registrada por Castro (1557), quien utilizaba las grafías $<a>y<a ̨>$ para representar la vocal [-densa] [-difusa] [bemolizada] /ə/. Por otro lado, considero que el registro de la vocal /o/ pudo ser un error gráfico, ya que solo está registrado por Andrews (1945), quien utilizaba las grafías $<\ddot{o}>$, $<\varnothing>$ y $<$ $>$ para la vocal /ə/. Lo considero como un posible error gráfico ya que solo utilizó una de aproximadamente 60 veces la grafía <0> en sus libretas de campo, caso en el que pudieron faltar las diéresis o la diagonal de las grafías utilizadas regularmente. Finalmente, el registro de la vocal /e/ podría deberse a la realización sostenida de la consonante aguda difusa glotalizada [t’y] del exponente, ya que la vocal se podría realizar como aguda [e] y no como aguda bemolizada [ə].

Los exponentes para la categoría de número eran ${ }^{*}-k^{w} e$ 'dual', *-ha 'plural' y para el singular se mantenía la posición estructural aunque no tenía un exponente afijal. Así, los exponentes de singular y dual eran los mismos que los presentados para los pronombres de primera y segunda personas $-ø \mathrm{y}-k^{w} e$. Los cambios fonológicos del sufijo de dual ${ }^{*}-k^{w} e$ son los ya descritos anteriormente, la lengua de San Juan Atzingo mantiene el segmento denso grave interrupto $[\mathrm{k}]$ mientras que el resto de las lenguas pierden 
dicho segmento y, por otro lado, agrega la secuencia /wi/ como parte de su estructura fonológica, resultando -wewi.

El sufijo de plural *-ha muy seguramente está relacionado con el sufijo de plural * $k^{h}$ a descrito para los pronombres de primera y segunda personas. Sin embargo en el paradigma de tercera persona no hay evidencia que permita considerar a la consonante densa grave $[\mathrm{k}]$ como parte de la estructura subyacente de este morfema en ninguna lengua, ni para reconstruirla para el protoatzinca. En este paradigma también se puede observar que el sufijo de plural no incorporó la secuencia final /wi/ como parte de su estructura fonológica, en ninguna de las lenguas. Este morfema de plural es el que se sufija a los sustantivos y verbos.

En las lenguas que tienen el exponente de tercera persona tawi se elide la vocal aguda difusa /i/ cuando se sufijan los morfemas de número. En el pronombre de tercera dual resulta una secuencia de dos consonantes idénticas /ww/ y en este caso se elide una de ellas (esquema 11), mientras que en los pronombres en plural la consonante aspirada del sufijo de número se realiza como rasgo de la consonante bemolizada, resultando $\left[\mathrm{w}^{\mathrm{h}}\right]$ (esquema 11).

Esquema 11. Cambios sincrónicos en los PPI de tercera dual y plural
a) [t'วwi-wewi $] \rightarrow$ [t’ow-wewi $] \rightarrow$ [t'owewi $]$

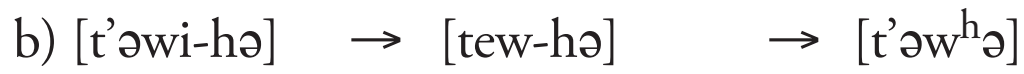

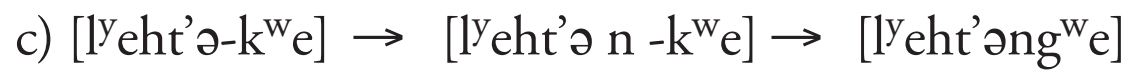

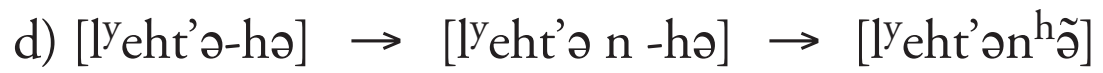

En la lengua de San Juan Atzingo, se inserta una consonante nasal/n/ en la frontera morfológica entre el exponente de tercera persona y el sufijo 
de número. Eso provoca que la consonante densa grave $[\mathrm{k}]$ del sufijo de dual, se realice como sonora [g] (esquema 11c). La consonante nasal también está registrada con el rasgo sostenido [ $\left.\mathrm{n}^{\mathrm{y}}\right]$, ya que, como mencioné anteriormente, las consonantes agudas difusas tienden a realizarse sostenidas en esta lengua (esquema d) y la vocal del sufijo de plural se realiza nasalizada.

El morfema * ${ }^{*}$ - ocupaba la posición más externa y se prefijaba al constituyente formado por el exponente de tercera persona y el sufijo de número. Por el momento, no tengo elementos para proponer a qué categoría pertenecía este morfema o cuál era su función. Propongo que era un prefijo, aunque es necesario contar con más datos de las lenguas derivadas para corroborarlo, ya que pudo tener otro estatus morfológico, como clítico por ejemplo.

El prefijo *re- tenía el templete CV y estaba formado por una consonante aguda difusa sonante que represento como */r/ y la vocal /e/ ([-densa] [-difusa] [+aguda]). No hay certeza para proponer si la consonante se realizaba como $*[\mathrm{r}],{ }^{*}[\mathrm{~d}] \mathrm{o} *[\mathrm{l}]$. Considero que probablemente no era lateral, en tanto que en las lenguas atzincas actuales, en el nivel fonológico, pertenece a la serie de localización de las agudas difusas y tiene el rasgo [+continuo] por ser fonéticamente fricativa. En la lengua de San Juan Atzingo la correspondencia de esta consonante es la líquida /l/ que tiende a palatalizarse al igual que el resto de las consonantes agudas difusas en esta lengua, a esto se debe que este registrada como [l] y [ly]. En el resto de las lenguas la correspondencia de esta consonante no es lateral y tiene dos alófonos [r] y [d]. En la tabla 11 de formas cognadas podemos obser- 
var que en las lenguas de Charo y Undameo el pronombre de tercera persona está registrado con la consonante $[\mathrm{d}]$.

Reconstruyo la vocal del exponente de tercera persona como corta */e/. Solo en la lengua de San Francisco Oxtotilpan se puede observar variación en la longitud de esta vocal. En la lengua de San Juan Atzingo tenemos la aspiración [h] en el reflejo de este prefijo [leh], llama la atención en tanto que la raíz pronominal para primera y segunda persona también se realiza con la aspiración [kah]. Es importante recordar que las vocales largas solo se presentan en las raíces. El hecho de que haya variación en la longitud de la vocal del exponente re-podría deberse a una tendencia de ajuste del templete morfológico de los pronombres en la lengua de San Francisco Oxtotilpan, esto recordando que en los pronombres de primera y segunda persona el elemento nuclear ocupa la primera posición de la palabra de izquierda a derecha y, por ser la raíz pronominal, es el que se presenta con vocales largas. Sin embargo, no es posible proponer que el morfema resea el núcleo estructural en tanto que el pronombre se puede realizar sin dicho elemento, como se puede observar en la tabla 6 de cognados en la lengua de San Francisco Oxtotilpan. Por otro lado, es claro que en la lengua de San Juan Atzingo la secuencia [leh] es parte del exponente de tercera persona, por lo tanto el ajuste no sería morfológico sino de la forma fonológica respecto de los pronombres de primera y segunda persona.

En la tabla 11 de pronombres de tercera se pueden observar otros morfemas al principio de palabra: we-, in-, $k i-, k a-y$ ta-. Propongo que estos afijos tienen una posición estructural distinta a la del prefijo re-, el cual solo se opone a su ausencia. En la lengua del Valle de Toluca el pronombre tiene el morfema ki-, en la lengua de Charo aparecen los morfemas 
$k i$ - in- y we-; en la lengua de Undameo observamos los morfemas $k i$ - e in-; finalmente la lengua de San Francisco Oxtotilpan encontramos los morfemas ta-, $k a-$ y $k i$-. En este última lengua no se permiten los morfemas in- y we-.

Los morfemas we-e in- en las lenguas coloniales y en la lengua de San Francisco Oxtotilpan se prefijan a los nominales; we- es empleado exclusivamente para ciertos términos humanos, mientras que in-se usa para no humanos y humanos. De acuerdo con Siewierska (2004) es posible que los pronombres tengan algunas características nominales y la tendencia es que los pronombres de tercera persona sean los que presenten más. De esta manera, es posible que los pronombres de tercera persona de las lenguas coloniales pudieran tener estos prefijos nominales, aunque esto ya no es posible en las lenguas actuales. En los pronombres de tercera plural de la tabla 11 se puede observar que los morfemas in- y we-coaparecen con el prefijo re-y la tendencia es que ocupen la primera posición de la palabra, esto indica que estos morfemas no tienen la misma posición estructural. La posición estructural de estos prefijos sería la más externa de la palabra pronominal. Sin embargo es necesario analizar su funcionamiento en textos para corroborarlo.

Castro (1557) registra para la lengua del Valle de Toluca el pronombre de tercera persona como [kit'awi] en las entradas 'él o ella, pronombre' y 'aquel, aquella, aquello'. Mientras que Guevara (1862[1638]), para Undameo, presenta el paradigma de pronombres de tercera como 'Del pronombre ille'; en singular registra [kit' ewi] 'aquel', en plural [int'ehwə] 'aquellos' y más adelante en una nota menciona el pronombre que corresponde al dual, [int'ewewi]. Los pronombres con el prefijo re-y con los 
morfemas we-y ki-presentados en la tabla 11, aparecen a lo largo del texto en paradigmas verbales y oraciones que presenta Guevara como ejemplos, traducidos indistintamente como 'él' o 'aquel' y sus correspondientes plurales. Por otro lado, Basalenque (1975a [1642]), para Charo, registra el paradigma de pronombres de tercera persona en el apartado de pronombres como 'aquel', en singular, dual y plural aparecen con el morfema in-. En la presentación de la letra $<\mathrm{d}>$ en la cartilla de las letras describe que esta letra se puede volver la letra $<\mathrm{r}>$ y ejemplifica con las formas [indegewi] y [kiregewi], explicando que "la $r$ en $d$ como quiregehui es $3^{\mathrm{a}}$. persona, fue hecho porque antecede in, si se hace nombre dice indegehui" (1975[1642]:126). El pronombre registrado como [det'ewi] aparece en el Vocabulario de la lengua matlazinga vuelto a la castellana, con el significado 'el mismo' (1975[1642]:183). A partir de estos datos, lo único que puedo proponer es que el prefijo re-no ocupaba la misma posición que los morfemas ki-, in- y we- en las lenguas coloniales. Las traducciones de los distintos registros del pronombre no aportan mayor información en tanto que posiblemente están más relacionadas con los usos del pronombre en castellano en la época colonial que con distinciones de significado en las lenguas atzincas.

En la lengua de San Francisco Oxtotilpan, el prefijo re-se opone a la ausencia del prefijo. Al elicitar el paradigma de pronombres, las formas que se obtienen tienen el prefijo re-y ningún elemento antes. Estas mismas formas son las que aparecen al elicitar paradigmas verbales con la tercera persona explícita con distintas funciones sintácticas. En los textos registrados por Cazés (1967), Escalante y Hernández (1999) y los míos, los pronombres tienen el prefijo re-. El pronombre sin el prefijo re- está registrado en los 
vocabularios de Escalante y Hernández (s.f. a y b). Por su parte, Carranza (2011) opina que el pronombre de tercera es [ret’owi] y la forma [t’owi] se usaría en un contexto de confusión como para aclarar 'sí, es él'. Al preguntar directamente por el pronombre sin el prefijo, me encontré con que ambas formas se traducen como 'él' y no hay una diferencia reconocida explícitamente, ya que no parece cambiar por la visibilidad o distancia del participante que refiere a tercera persona respecto de los que refieren a primera y segunda en el hecho discursivo. Por el momento, considero que no es posible atribuir de forma clara un significado o función a este prefijo. Hace falta tener más datos y pruebas de distintos tipos.

Respecto a los morfemas $k i$-, $t a-$ y $k a-$ en la lengua de San Francisco Oxtotilpan, considero que tienen una posición estructural distinta a la del prefijo re-. Esto aun cuando no se puede observar que coaparecen en los datos. Es probable que los morfemas $k i$-, $k a$ - y $t a$ - pertenezcan al ámbito verbal y ante el pronombre de tercera formen significados como 'es él' en tanto que los encontramos en construcciones de este tipo, registradas por Escalante y Hernández (1999), las cuales se pueden observar en los ejemplos de 1 a 3.

(1) tašuwi

ta-šuwi

TA-mujer ${ }^{9}$

'Soy mujer'

9 TA: tiempo y aspecto, D: determinante, 3: tercera, Ps: posesivo, s: singular. 
(2) inwhán kinmaestro

in-whán ki-n-maestro

D-Juan TA-?-maestro

'Juan es maestro'

(3) nišú kan Pédro

ni-šú ka-n-Pédro

3.PS.s-nombre TA -?-Pedro

'Tu nombre es Pedro'

En los tres ejemplos anteriores los morfemas $k i-, k a-y$ ta- anteceden a sustantivos y forman construcciones verbales. Estos morfemas marcan oposiciones de tiempo y aspecto (TA). Es necesario contar con más datos en discurso y verificar si estos morfemas pueden anteceder a los PPI de primera y segunda persona. Es importante no perder de vista que el morfema $k a$ - tiene la misma forma fonológica que la raíz pronominal de los pronombres de primera y segunda persona. Sin embargo, por el momento, no es posible proponer que se trate del mismo exponente, aunque es posible que tengan el mismo origen histórico pero en un estado de lengua anterior.

\section{Consideraciones finales}

La comparación y reconstrucción de las categorías gramaticales expresadas en los pronombres mostró que no hay cambios del protoatzinca hacia las lenguas derivadas. Por otro lado, en la comparación y reconstrucción de 
los exponentes morfológicos sí se observaron cambios que permiten agrupar las lenguas por las diferencias que generaron. Los cambios son de varios tipos: a) estructura de palabra morfológica; b) forma fonológica subyacente de los exponentes; c) restricciones y reajuste táctico; y d) reglas alofónicas. En el esquema 12 presento los tipos de cambio por lengua. En la primera columna presento el tipo de cambio (a, b, c, d) que generó las diferencias, en la segunda columna, el elemento comparado y en las columnas de la derecha presento cómo se realiza dicho elemento en cada lengua. En algunos casos indico si se permite o no tal elemento, o si no hay información suficiente (indicado con <?>). En este esquema no incluyo la lengua etiquetada como matlatzinca en las tablas de formas cognadas anteriores, en tanto que se trata de las lenguas de San Francisco Oxtotilpan y Mexicaltzingo. En todo caso, podemos suponer que estas lenguas eran muy cercanas, ya que Hasler (1961) y Soustelle (1993[1937]) no separaron los datos.

Esquema 12. Comparación de rasgos morfológicos, fonológicos y fonéticos

\begin{tabular}{|c|c|c|c|c|c|c|c|}
\hline $\begin{array}{l}\text { Tipo de } \\
\text { cambio }\end{array}$ & Elemento comparado & VT* & Und & Charo & Mex & Oxtotilpan & \multirow{2}{*}{\begin{tabular}{|r|} 
Atzingo \\
let’o
\end{tabular}} \\
\hline \multirow[t]{2}{*}{$\mathrm{a}$} & Exponente de tercera persona & \multicolumn{5}{|l|}{ t’awi } & \\
\hline & Prefijos nominales we- $\mathrm{y}$ in- & $?$ & \multicolumn{4}{|c|}{\begin{tabular}{|l|l|} 
sí se permiten & no se $\mathrm{p}$ \\
\end{tabular}} & \\
\hline \multirow[t]{5}{*}{$\mathrm{b}$} & $\begin{array}{l}\text { Secuencia /wi/ en los } \\
\text { exponentes de tercera } \\
\text { persona, dual y plural }\end{array}$ & \multicolumn{5}{|c|}{ sí } & no \\
\hline & Dual & \multicolumn{5}{|c|}{-wewi } & $-k^{w} e$ \\
\hline & $\begin{array}{l}\text { Plural para primera y } \\
\text { segunda persona }\end{array}$ & \multicolumn{5}{|c|}{-howi } & $-k^{h} o$ \\
\hline & $\begin{array}{l}\text { Fonema difuso, agudo, } \\
\text { continuo }\end{array}$ & \multicolumn{5}{|c|}{ vibrante $/ \mathrm{r} /$} & líquido /1/ \\
\hline & $\begin{array}{l}\text { Longitud de la vocal de la } \\
\text { raíz en segunda persona }\end{array}$ & \multicolumn{3}{|c|}{ corta } & $?$ & \multicolumn{2}{|c|}{ larga } \\
\hline
\end{tabular}




\begin{tabular}{|c|c|c|c|c|c|c|c|}
\hline $\begin{array}{l}\text { Tipo de } \\
\text { cambio }\end{array}$ & Elemento comparado & $\mathbf{V T}^{*}$ & Und & Charo & Mex & Oxtotilpan & Atzingo \\
\hline \multirow[t]{3}{*}{ c } & $\begin{array}{l}\text { Presencia de /n/ en la } \\
\text { frontera morfológica de } \\
\text { tercera persona y plural }\end{array}$ & \multicolumn{5}{|c|}{ no se presenta } & $\begin{array}{c}\text { sí se } \\
\text { presenta }\end{array}$ \\
\hline & $\begin{array}{l}\text { Elisión de la vocal /i/ de los } \\
\text { exponentes de persona ante } \\
\text { los de número }\end{array}$ & \multicolumn{5}{|c|}{ en primera y segunda persona } & $\begin{array}{l}\text { solo en } \\
\text { primera } \\
\text { persona }\end{array}$ \\
\hline & $\begin{array}{l}\text { Procesos para romper grupos } \\
\text { consonánticos }\end{array}$ & \multicolumn{5}{|c|}{$\begin{array}{l}\text { consonante del morfema de número } \\
\text { como rasgo de la consonante del } \\
\text { morfema de persona }\end{array}$} & $\begin{array}{c}\text { elisión de } \\
\text { consonantes } \\
\text { iguales }\end{array}$ \\
\hline \multirow[t]{3}{*}{$\mathrm{d}$} & $\begin{array}{l}\text { Alófonos sostenidos de las } \\
\text { consonantes agudas }\end{array}$ & \multicolumn{5}{|c|}{ no } & $\begin{array}{c}{\left[\mathrm{I}^{\mathrm{y}}\right],\left[\mathrm{t}^{\mathrm{y}}\right]} \\
{\left[\mathrm{n}^{\mathrm{hy}}\right]}\end{array}$ \\
\hline & $\begin{array}{l}\text { Sonorización de la } \\
\text { consonante inicial /k/ }\end{array}$ & \multicolumn{5}{|c|}{$[\mathrm{g}]$} & $?$ \\
\hline & $\begin{array}{l}\text { Vocal final de los pronombres } \\
\text { de primera singular }\end{array}$ & \multicolumn{5}{|c|}{ [i] } & [ə] \\
\hline
\end{tabular}

* VT: Valle de Toluca, Und: Undameo, Mex: Mexicaltzingo.

Los cambios en la estructura de palabra morfológica (a) los encontramos solamente en los pronombres de tercera persona. A partir de estos cambios tenemos, por un lado, dos exponentes de tercera persona distintos let’o en la lengua de San Juan Atzingo y t’awi en el resto de las lenguas. Y por otro lado, tenemos que en las lenguas de Undameo y Charo sí se permiten los prefijos we- y in-, mientras que en los actuales no se permiten.

Los cambios en la forma fonológica subyacente de los exponentes (b), las restricciones y reajuste táctico (c), y las reglas alofónicas (d), generaron diferencias que en su mayoría separan a la lengua de San Juan Atzingo del resto. Las dos excepciones son la longitud de la vocal de la raíz en los pronombres de segunda persona, ya que la tendencia es que sea larga en las lenguas actuales y no en las coloniales, aunque no hay suficientes datos para 
la lengua de Mexicaltzingo. La otra diferencia es la sonorización de la consonante inicial de los pronombres de primera y segunda persona, que solo está registrada en las lenguas de Mexicaltzingo y San Francisco Oxtotilpan.

Así pues, la comparación de los elementos mostrados en el esquema 12 presenta diez diferencias que distinguen a la lengua de San Juan Atzingo del resto y tres que distinguen a las lenguas coloniales de las actuales. Sin embargo, considero que, para comprender de forma más adecuada el desarrollo histórico de las lenguas atzincas, es necesario reconstruir de forma integral otros sistemas y subsistemas de la lengua. En este sentido, la reconstrucción y descripción que aquí presenté no pretenden proponer una separación histórica entre la lengua de San Juan Atzingo, sino que se suman a los aportes de Bartholomew (1965), Hasler (1961), Valiñas (2000) y Pérez (2007), entre otros, para conocer las relaciones entre las lenguas atzincas y la gramática del protoatzinca.

\section{Bibliografía}

Aguilar, Yásnaya y Leopoldo Valińas, 2008. "Sistema de pronombres personales libres en algunas lenguas nacionales mexicanas." Ponencia presentada en el VII Coloquio de Lingüistica en la Escuela Nacional de Antropológica e Historia, México, Escuela Nacional de Antropología e Historia.

Andrews, Henrietta, 1945. Libretas de campo II y III. Ms.

Andrews, Henrietta, s.f. Observaciones en fenómenos tonales de matlatzinca. Ms. Andrews, Henrietta y Olive Shell, s.f. A Preliminary Report on Matlatzinca Grammar. Ms. 
Bartholomew, Doris. 1964. Vocabulario de San Juan Atzingo, 3 de septiembre de 1964. Ms.

Bartholomew, Doris, 1965. The reconstructon of otopamean (Mexico). Tesis Doctoral. Chicago: The University of Chicago.

Bartholomew, Doris. 1970. Observations about Matlatzinca Grammar based on informant work done in May, 1966. Ms.

Bartholomew, Doris, 1992. "Reverberaciones de la pérdida de la $h$ en la transcripción del matlatzinca en los siglos xvi y xvıı". En Elizabeth Luna Traill (coord). Scripta Philologica in Honorem Juan M. Lope Blanch. México: Universidad Nacional Autónoma de México, Vol. 3, pp. 17-37.

Bartholomew, Doris, s.f. Fichero, pronombres. Ms.

Basalenque, Diego, 1975a [1642]. Arte y Vocabulario de la lengua matlatzinca vuelto a la castellana. Versión paleográfica de María Elena Bribiesca S., estudio preliminar de Leonardo Manrique. Toluca: Biblioteca Enciclopédica del Estado de México.

Basalenque, Diego, 1975b [1642]. Vocabulario de la lengua castellana vuelto a la matlatzinca. Revisión paleográfica, nota introductoria y apéndice por Leonardo Manrique. Toluca: Biblioteca Enciclopédica del Estado de México.

Benveniste, Emile. 1971[1966]. Problemas de Lingüistica General 1. México: Siglo XXI.

Carranza, Leonardo, 2011. Paradigma de pronombres y notas. Ms.

Castro, Andrés, 1557. Vocabularo castellano-matlatzinca. Versión paleografiada por Doris Bartholomew. Ms.

Cazés, Daniel, 1977. "Glotocronología hña-mclasinka-meco(otopame)" en Amerindia. Revue D'Ethnolinguistique Amerindienne 2, pp. 65-115. 
Cazés, Daniel, 1967. El Pueblo Matlatzinca de San Francisco Oxtotilpan y su Lengua. Acta Anthropologica, Segunda Época 2(3).

Escalante, Roberto y Marciano Hernández, 1999. Matlatzinca de San Francisco Oxtotilpan, Estado de México. Archivo de lenguas indígenas de México 23. México: El Colegio de México.

Escalante, Roberto y Marciano Hernández, s.f.a. Vocabulario español-matlatzinca. Fichero Personal. México: Dirección de Lingüística. Instituto Nacional de Lenguas Indígenas.

Escalante, Roberto y Marciano Hernández, s.f. b. Vocabulario español-matlatzinca. Ms.

García Payón, José, 1936. La Zona Arqueológica de Tecaxic-Calixtlabuaca y los Matlatzincas. México: Talleres Gráficos de la Nación.

Guevara, Miguel, 1862 [1638]. "Arte doctrinal y modo general para aprender la lengua matlatzinca”. En: Boletin de la Sociedad Mexicana de Geografía y Estadística. México: Sociedad Mexicana de Geografía y Estadística. Hasler, Juan A., 1961. "Reconstrucciones matlatzinca-ocuiltecas". Anales XIII, 42, México: Instituto Nacional de Antropología e Historia, pp: 269-278.

Jakobson, Roman, 1981 [1957]. "Los conmutadores verbales, las categorías verbales y el verbo ruso". En Ensayos de lingüistica general. Barcelona: Seix Barral, pp. 307-332.

Jakobson, Roman y Linda R. Waugh, 1979. La forma sonora de la lengua. México: Fondo de Cultura Económica.

Koch, Nancy L., 2000. Preliminary Grammar of Matlatzinka, ms.

Meléndez, Lucero, 2011. El sistema de persona del proto-huasteco. Tesis de Doctorado. México: Universidad Nacional Autónoma de México. 
Muntzel, Martha C., 1986. The Structure of Ocuilteco. Tesis de Doctorado. Albany: University of New York at Albany.

Pascacio, Etna, 2006. Fonología de la lengua matlatzinca de San Francisco Oxtotilpan. Tesis de Licenciatura. México: Escuela Nacional de Antropología e Historia.

Pascacio, Etna, 2011. Pronombres personales independientes del proto-atzinca: reconstrucción morfológica. Tesis de Maestría. México: Universidad Nacional Autónoma de México.

Pérez, Nancy, 2007. Synchronic and Diachronic Matlatzinkan Phonology. Tesis de Doctorado. The University of Michigan.

Reynoso González, Elpidia, 1998. Vocabulario español-tlabuica. Toluca: Instituto Mexiquense de Cultura, Gobierno del Estado de México.

Schumann G., Otto, 1975. "Notas sobre la lengua ocuilteca y sus relaciones”. En: Roman Piña Chan (ed.). Teotenango: el antiguo lugar de la muralla. Memoria de las Excavaciones Arqueológicas, México: Dirección de Turismo del Gobierno del Estado de México, Vol. 2, pp. 529-539. Siewierska, Anna, 2004. Person. Cambridge: Cambridge University Press. Sin autor, 1997. Diccionario Matlatzinca-Español. Estado de Toluca: Colegio de Lenguas y Literatura Indígenas.

Soustelle, Jaques, 1993 [1937]. La familia otomi-pame del México central. México: Fondo de Cultura Económica.

Trubetzkoy, Nicolái, 1973[1939]. Principios de Fonología. Madrid: Cincel. Valiñas C. Leopoldo, 2000. "El matlatzinca y el ocuilteco, ¿eran ya lenguas distintas en el siglo xvi?”. En Estudios de cultura otopame. México: Instituto de Investigaciones Antropológicas, Universidad Nacional Autónoma de México, pp. 73-96.

Weitlaner, Roberto, s.f. Vocabulario español-matlatzinca. Ms. 
Pronombres personales del protoatzinca

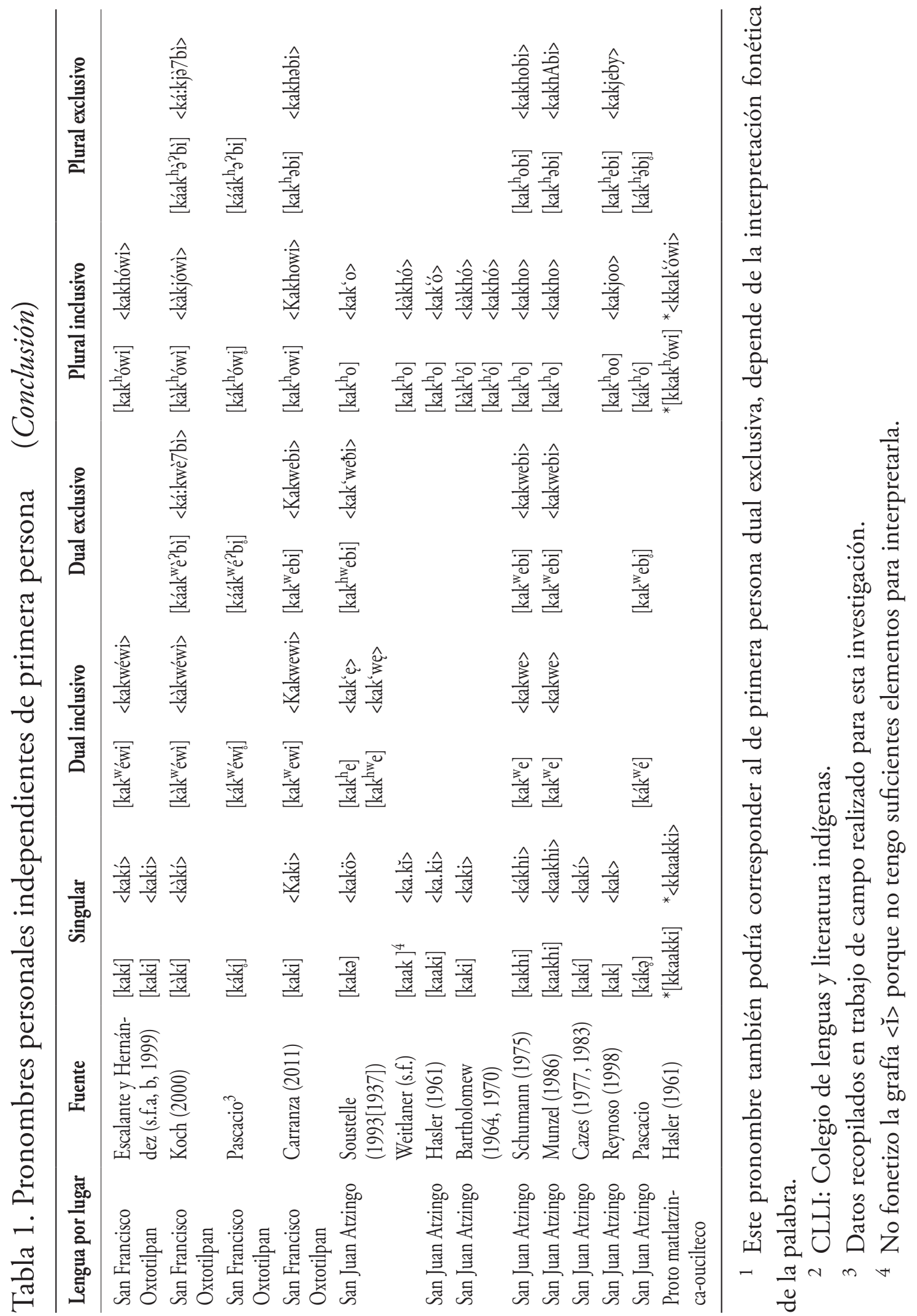




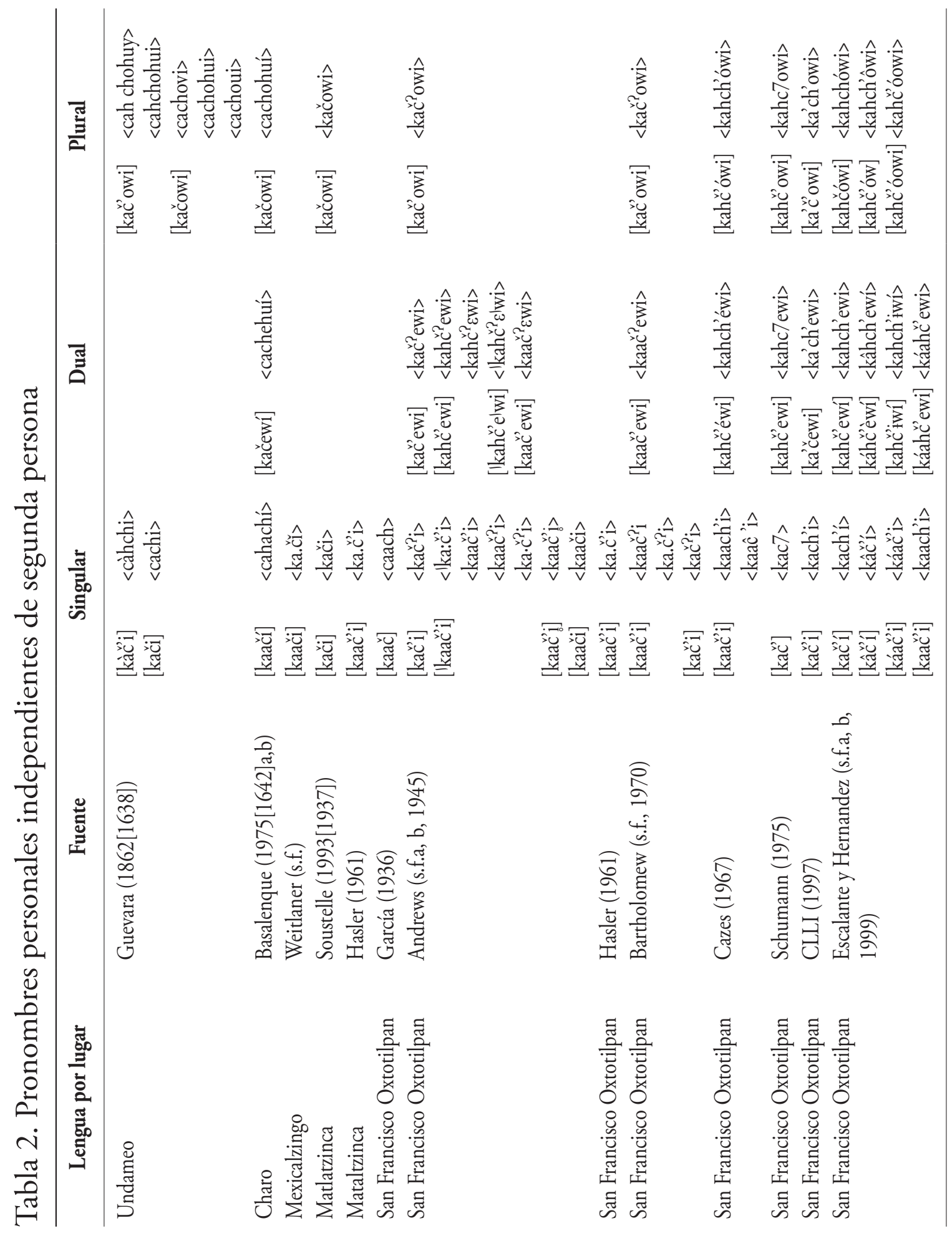




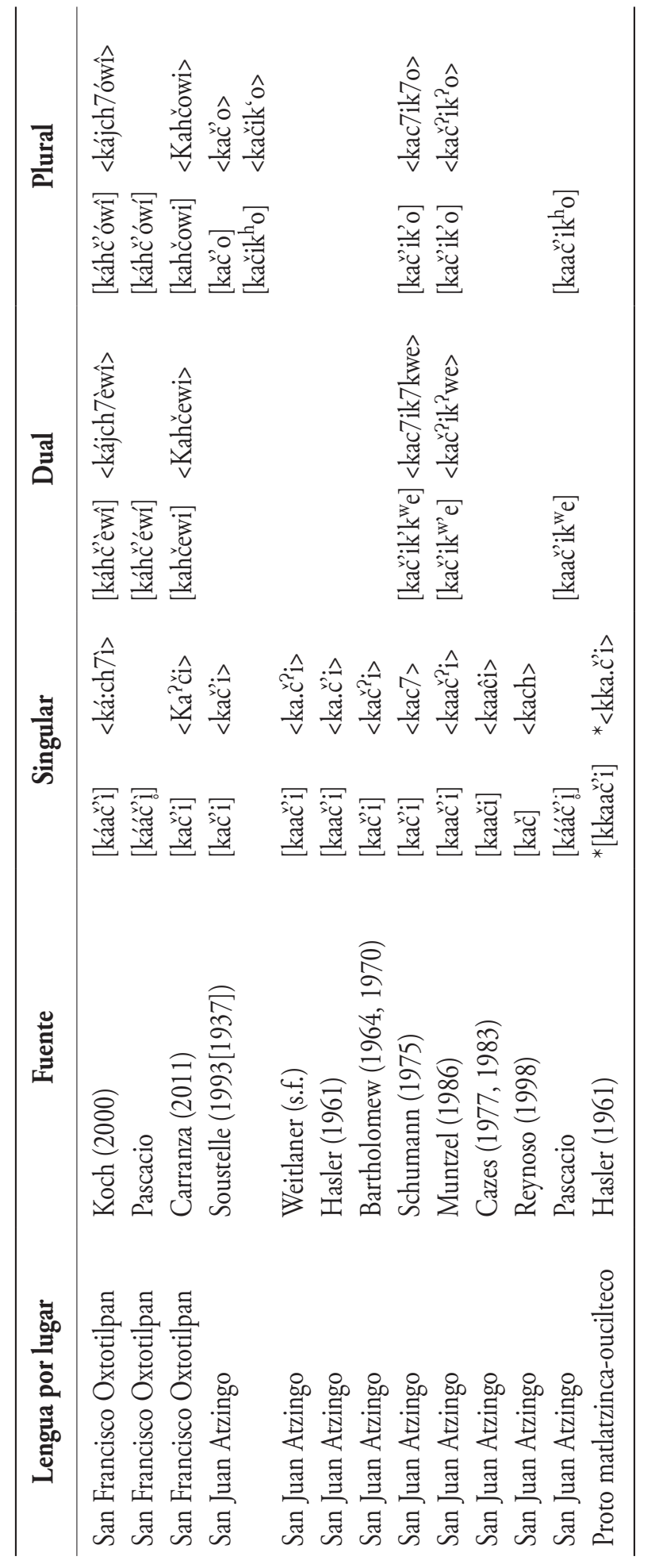




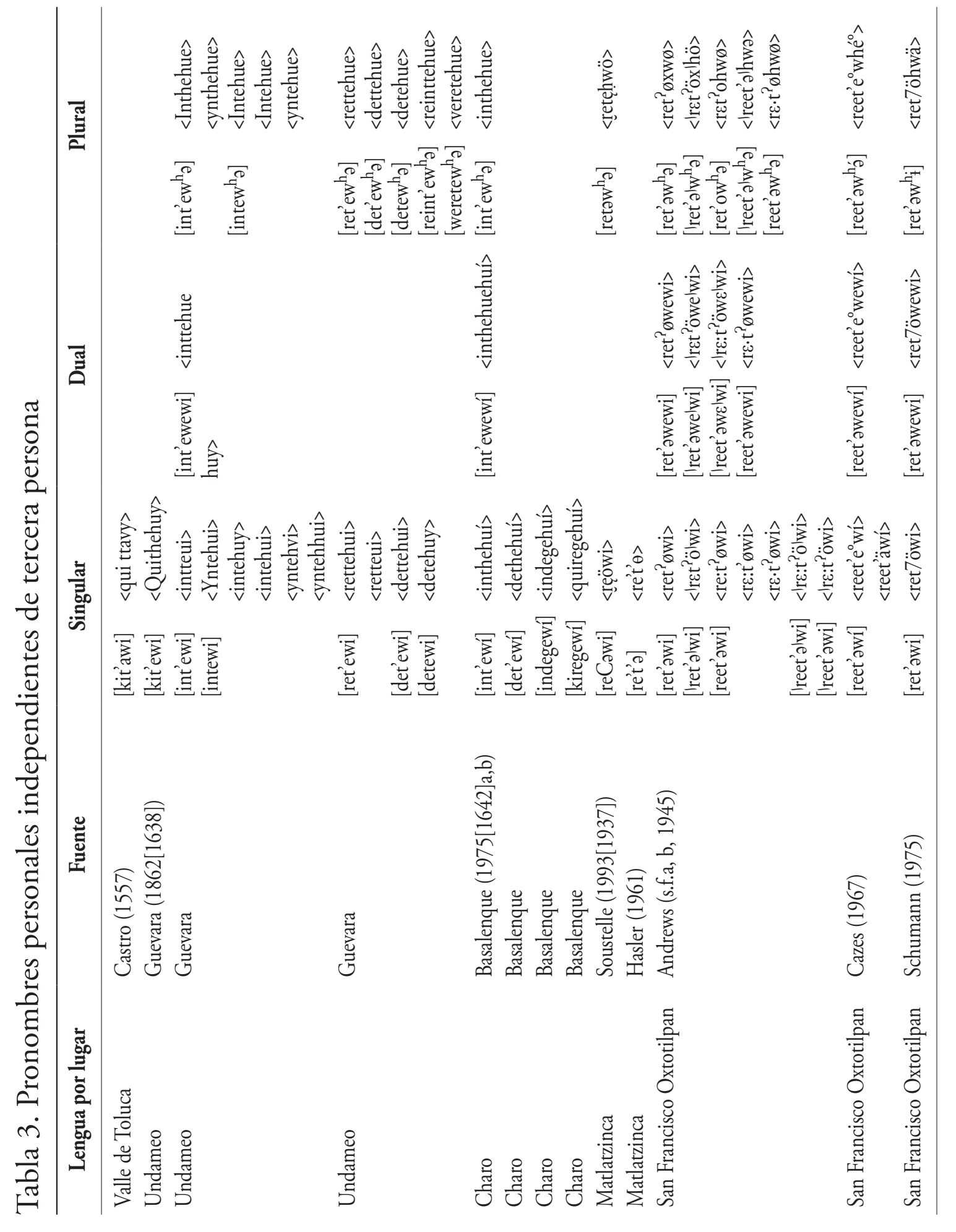


Pronombres PERSONALES DEL PROTOATZINCA

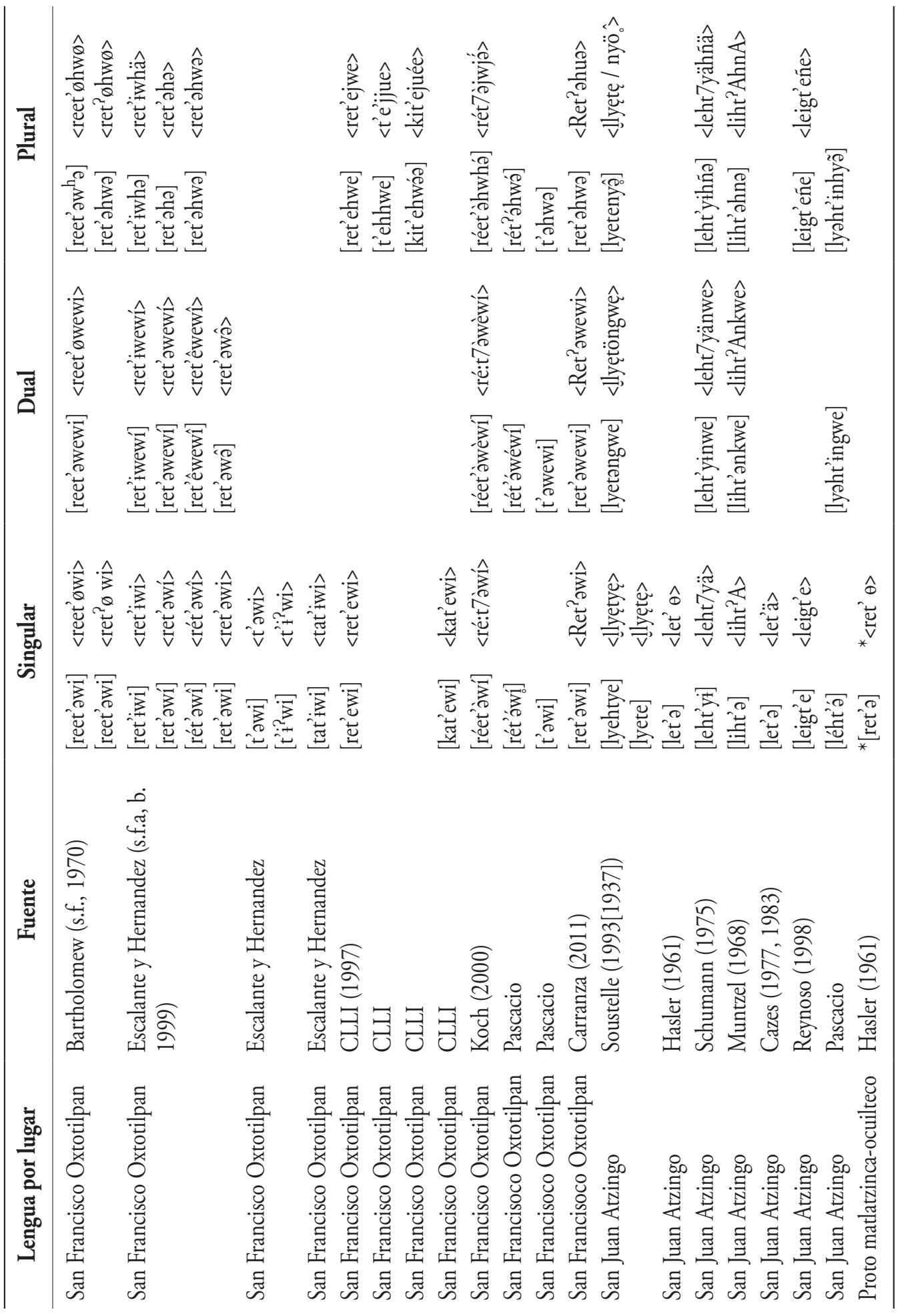

